BANCA D'ITALIA

E U R O S I S T E M A

Questioni di Economia e Finanza

(Occasional Papers)

A game changer in payment habits: evidence from daily data during a pandemic

by Guerino Ardizzi, Andrea Nobili and Giorgia Rocco

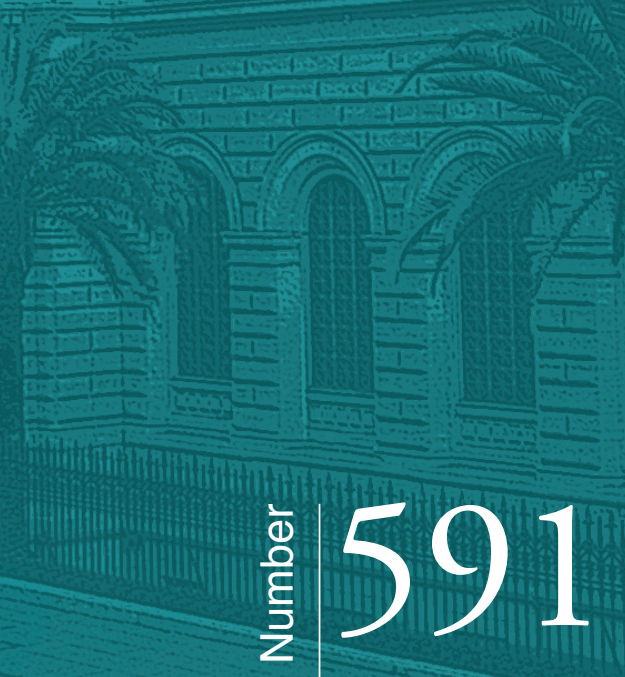



13 BANCA D'ITALIA

E U ROS IS T E MA

\section{Questioni di Economia e Finanza}

(Occasional Papers)

A game changer in payment habits:

evidence from daily data during a pandemic

by Guerino Ardizzi, Andrea Nobili and Giorgia Rocco

Number 591 - December 2020 
The series Occasional Papers presents studies and documents on issues pertaining to the institutional tasks of the Bank of Italy and the Eurosystem. The Occasional Papers appear alongside the Working Papers series which are specifically aimed at providing original contributions to economic research.

The Occasional Papers include studies conducted within the Bank of Italy, sometimes in cooperation with the Eurosystem or other institutions. The views expressed in the studies are those of the authors and do not involve the responsibility of the institutions to which they belong.

The series is available online at www.bancaditalia.it.

ISSN $1972-6627$ (print)

ISSN 1972-6643 (online)

Printed by the Printing and Publishing Division of the Bank of Italy 


\title{
A GAME CHANGER IN PAYMENT HABITS: EVIDENCE FROM DAILY DATA DURING A PANDEMIC
}

\author{
by Guerino Ardizzi*, Andrea Nobili* and Giorgia Rocco*
}

\begin{abstract}
We explore the relationship between cash and other payment instruments using the outbreak of the COVID-19 pandemic as a natural experiment exogenously affecting both the payment industry and consumers' habits. We rely on Google search data, as well as on the official series of new cases of infection to measure the intensity of the pandemic, and apply local projection methods to assess the effects on payment habits. We find a large and persistent substitution effect from cash to card-based transactions, especially using contactless and ecommerce options. The fear of infection has led to a new implicit cost associated to each payment instrument, thus affecting payment choices from the demand-side and boosting consumption with non-cash transactions. Moreover, technical constraints on the cash cycle and the lockdown measures have increased the demand for cash for precautionary purposes. Policies aiming at accelerating the digital economy and the most innovative means of payment can potentially make economic activity more resilient to adverse shocks. At the same time, ensuring the adequate and efficient availability of cash remains essential from a social and economic perspective.
\end{abstract}

JEL Classification: E41, E42, G2, O3

Keywords: COVID-19 pandemic, cash, payment habits, unconventional data

DOI: $10.32057 / 0 . Q E F .2020 .591$

\section{Contents}

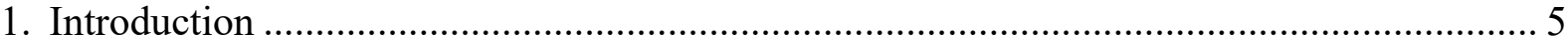

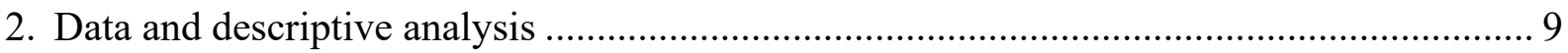

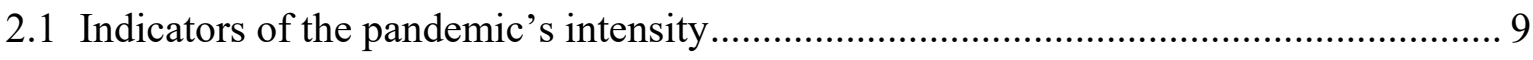

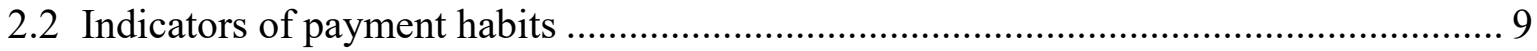

3. A primer on the effects of the pandemic on payment habits ............................................ 12

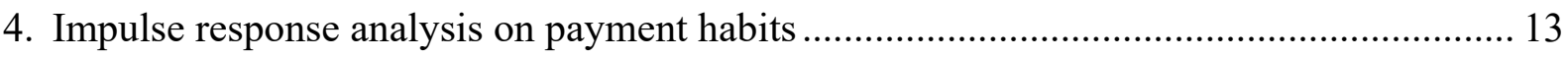

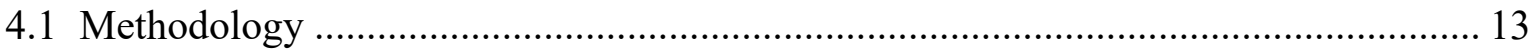

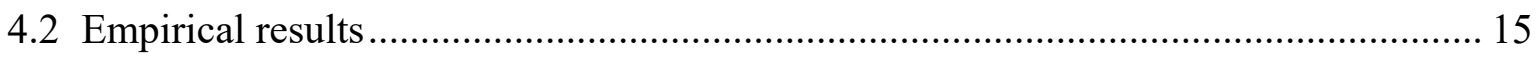

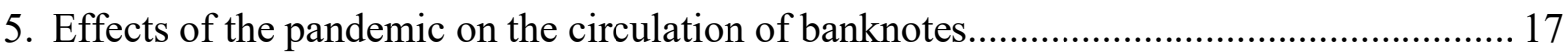

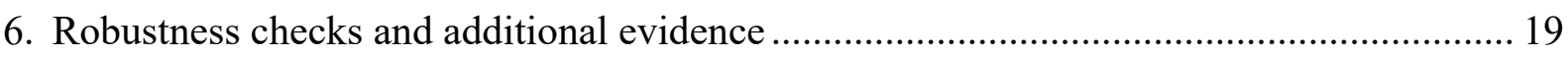

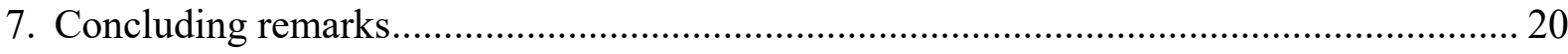

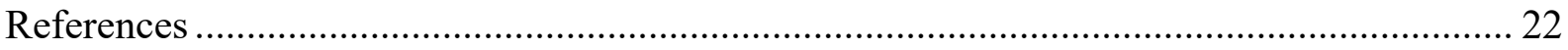

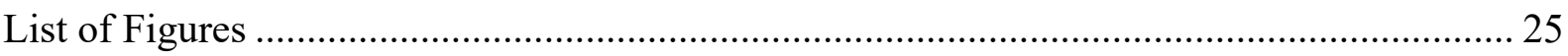

Appendix A. Effects of the pandemic on other variables ................................................ 32

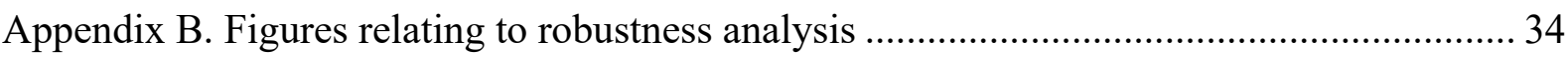

* Bank of Italy, Directorate General for Currency Circulation and Retail Payments. 



\section{Introduction ${ }^{1}$}

The empirical relationship between cash and innovations in retail payments technology is at the heart of the current policy debate. A global transformation of the payment industry has been underway for several years, challenging incumbent providers and re-shaping long-established relationships, timescales, and payment methods. The widespread adoption of debit and credit cards, the innovations in online-banking, the new payment technologies such as contactless, mobile and instant payments, the activity of "Fintech" and large internet platform companies (Google, Facebook, Amazon), nowadays represent an alternative to cash.

Besides these on-going trends in the industry of payment services, the outbreak of the COVID-19 pandemic since early 2020 represented an unprecedented shock for the global economy and dramatically affected firms and consumers' behaviors, including their social and payment habits. The fear of infection has prompted retailers to ask customers to wear masks and maintain physical distancing, and customers on their sides have preferred to avoid any contact, even when it comes to the act of paying. Despite scientific evidence indicating that banknotes are probably not a major channel of infection (Panetta, 2020; Auer et al., 2020 and references therein), there was an exceptional weariness around the use of cash, thus dramatically accelerating the rate of adoption of digital banking services, including payments and contactless instruments.

In retail trade, the upward trend in the use of digital sales channels and online purchases with electronic payments has suddenly intensified (Visco, 2020). Many economists argue that this sanitary emergency can be a great opportunity to accelerate the transformation of the economy and to endorse policies that limit cash usage to fight several illegal activities that are known to be cash-intensive (Rogoff, 2016). Whereas end-users usually perceive cash for payment as free of charge, survey data on the social cost of different payment methods indicate that cash is an expensive instrument for society and gradual migration to electronic instruments leads to an overall saving of resources (Bank of Italy, 2012, 2020). Market experts believe that the pandemic is a "game-changer" in the process of substitution of cash and other traditional means of payment in society, pushing the adoption of the most innovative payment solutions in business models of merchants. ${ }^{2}$

Understanding how the outbreak of the pandemic affected the retail payment industry and the demand for cash and other means of payment is, therefore, of first-order interest for central banks in order to take their policy and strategic decisions and to ensure the smooth functioning of the payment system. In this regard, this paper addresses a number of relevant questions. What about the effects of the COVID-19

\footnotetext{
${ }^{1}$ We are grateful to Marco Bernardini, Massimo Doria, Paola Giucca, Gianluca Maddaloni, Michele Manna, Gianmatteo Piazza, Ferdinando Sasso and Stefano Siviero for their insightful suggestions and remarks on a previous draft of this paper. We are also grateful to Alessandro Gambini for suggestions on the interpretation of BI-COMP data. Emanuele Pimpini provided invaluable help with the data. The views expressed herein are those of the authors and do not necessarily reflect those of the Bank of Italy. All the remaining errors are ours.

2 The transition towards a cashless society can imply restrictions to access to payment services in some
social classes and difficulty to rely on safe and liquid assets in times of financial distress. For these
reasons, central banks are today evaluating electronic cash substitutes, namely the design and the
implementation of a central bank digital currency (European Central Bank, 2020). Currently, cash is the
only legal tender, easy to use and accessible to all, including those people who do not have a bank account.
Cash is not only used as payment instruments, as its "safe-haven" role played in the economy in periods
of uncertainty or financial strains has been discussed in Stix (2013), Fisher et al. (2004), Rinaldi (2017),
Jobst and Stix (2017), Bech et al. (2018).
} 
pandemic on payment habits? Are these effects persistent? If yes, are we moving faster towards a cashless society?

We investigate the dynamic effects of the pandemic on payment habits using a unique dataset available at a daily frequency. We rely on unconventional data from Google search queries involving keywords relating to the pandemic and payment instruments to capture the fear of infection and to build up a measure of the pandemic intensity. We compare developments in this indicator and its effects on payment habits with those stemming from the official daily time series of newly infected people provided by media. The latter can capture other mechanisms at work - not strictly related to the fear of infection - that may affect payment choices. The lockdown measures to limit the infection, indeed, implied a sudden change in households' consumption basket with a switch from services and non-essential goods towards food and medical products, as well as a strong decline in the frequency of purchases. Moreover, the closing of many small non-essential activities and the negative effects of the severe recession on employment and disposable income may have tightened both the budget and borrowing constraints inducing people to use credit cards instead of cash and debit cards.

As regard indicators of payment habits, we consider high frequency and confidential data available at the Bank of Italy. We construct indicators of preference for cash against electronic payments at the point-of-sale (the so-called cash-card ratio), variables based on ATM withdrawals that play a role in inventory models of cash demand, measures of usage of the most innovative instruments - notably contactless cards - and the share of payments made on e-commerce websites. Finally, we also explore the effects on banknotes in circulation for different denomination notes to corroborate the view that the pandemic led to a reduction of cash for transaction purposes, as opposed to an increase in the precautionary demand for cash.

Besides the quality and granularity of our dataset, we improve upon previous literature on payment habits in several aspects. Firstly, we shed light on the nexus between cash and other payment instruments in Italy using the outbreak of the COVID19 pandemic as a natural experiment exogenously affecting both the payment industry and consumers' payment choices. In this regard, we assess whether payment choices are complements or substitutes conditionally to an unpredictable event hitting the economy. While it is very unlikely that transactions affect the news on the pandemic, the application to daily data largely avoids any other endogeneity concern, that we consider negligible within a day but potentially relevant with monthly or quarterly data.

Secondly, the outbreak of the pandemic may have introduced a new "implicit" cost associated to the different fear of infection when using payment instruments, thus inducing a pecking order in the payment choices. This cost acts from the demand-side of the payment market, is not directly measurable but it has the same economic implications of other types of monetary costs proposed by the empirical literature. These are opportunity, transaction and handing costs (Klee, 2008), fees on credit cards (Borzekowski et al., 2008), as well as credit card rewards, which essentially represent a negative cost on such instruments and imply re-composition effects in favour of electronic payments (Simon et al., 2010; Ching and Hayashi, 2010; Arango et al., 2015). At the same time, the fear of infection represents a kind of "security cost". In previous studies, the consumer perception of security is a significant factor affecting payment habits and private consumption (Kosse, 2013; Schuh and Stavins, 2015; Greene and Stavins, 2017; Kahn et al., 2017; Ardizzi et al., 2019) but it relates to the fear of frauds and thefts. 
Thirdly, the joint use of daily data on both cash and other payment instruments offers empirical evidence to enrich theoretical models involving technological changes and cash demand (Alvarez and Lippi, 2009; Lippi and Secchi, 2009). Recently, Alvarez and Lippi (2017) provided a theoretical setup that integrates the payment instrument choice into an inventory model of cash-management in which a representative agent chooses to pay with either cash or credit at each point in time, depending on the stock of cash-in-hand. Our analysis does not strictly focus on payment habits as a function of the stock holdings but is conceptually interesting because it considers outcome variables related to inventory models, notably the average amount of ATM withdrawals. The effect of the pandemic on such variable is a priori ambiguous. The preference for noncash payments induced by the fear of infection should imply that cash usage becomes less frequent, even for low-value transactions, thus leading to a decline in this indicator. On the other hand, the mobility restrictions induced by the lockdown could have induced people to increase their average withdrawal at ATM.

The substitution effects between cash and card usage and the predictions of inventory models have been also addressed by Brown et al. (2020) using administrative data. Differently from our paper, the authors focus on the effects of the introduction of contactless technology, which affect the usage of payment instruments from the supplyside. They provide evidence of increasing use of debit cards at POS, especially for smallvalue payments made by young consumers in urban locations, but a moderate reduction in the cash share of payments and no significant effect on the frequency and average size of cash withdrawals. ${ }^{3}$

Another important contribution is that we not only measure the sign and the magnitude of the pandemic impact but also evaluate the persistence of the effects over time, which is relevant for the related policy implications. We apply local projection techniques (LP, hereafter) proposed by Jorda (2005), which is particularly appealing in our context. This method, indeed, relies on simple regressions in which we identify the exogenous factor affecting the payment industry outside the data generating process of the outcome variables. Moreover, the resulting estimates with daily data are more robust to model misspecification and omitted variable problems arising when dealing with lowfrequency data. Finally, LP methods allow straightforward simulations with nonlinear and flexible models. In this regard, we can compare the effects of the pandemic on payment habits in sample periods characterized by large differences in the perception of the risk infection, in the restrictive measures and their impact on the economy. Our paper, therefore, differs considerably in the methodology from most of the previous studies on payment habits that relied on micro data, especially from surveys and diaries, which took an instant picture of the payment instruments using discrete choice models. ${ }^{4}$

\footnotetext{
3 As for the role of technological innovation, Rysman and Schuh (2017) described prospects and impediments to adoption of mobile and faster payments, as well as digital currencies. Among supply-side factors affecting payment habits, the literature on survey data also discussed the role played by merchants' acceptance of card at the point of sale (Arango et al., 2015; Wakamori and Welte, 2017). In this regard, promoting market competition and lowering the "interchange fee" that merchants pay for accepting credit and debit card payments can led to higher acceptance of electronic payment instruments, as shown in Li et al. (2019), Ardizzi and Savini Zangrandi (2018).

${ }^{4}$ The empirical literature using micro data from survey and diary data is wide. Stavins (2017) discussed supply and demand factors affecting payment behavior in the United States and summarized existing evidence for other countries, as well. For a cross-country analysis, see Bagnall et al. (2016). Evidence for Italy is discussed in Rocco (2019) and Ardizzi et al. (2020).
} 
We find that the pandemic induced significant substitution effects in payment choices. Consumers reduced the use of cash in transactions while increased the usage of cards at POS, especially those based on contactless technology. At the same time, we find a higher recourse to e-commerce purchases. Interestingly, most of these effects are persistent over time and not confined to the lockdown period, when the fear of infection was exceptionally high. We also corroborate the evidence of a decline in money holdings for transaction purposes by looking at the response of low-denomination banknotes, which is indeed negative. High- and medium-denomination notes instead increased, in relation to an impairment in the cash cycle during the pandemic, as well as to the role played by cash demand for precautionary motives. The results of a recent survey conducted by the ECB on the impact of the pandemic on cash trends (i.e. the IMPACT survey) support these figures, confirming that Italian consumers use less cash than before the pandemic as well as they declare that they would certainly maintain this attitude when the crisis will be over.

We also relate to empirical studies using card transaction data to track the shortterm pattern of consumption in advance of the official release of national statistics. ${ }^{5}$ Recent contributions documented interesting aggregate and sectoral dynamics in consumption during the most acute phase of the pandemic using a dummy-variable approach (Bounie et al., 2020; Carvalho et al., 2020; Chen et al., 2020). In the paper, we show that the substitution effect in favor of contactless cards is even stronger for purchases in the grocery sector, less affected by the lockdown and usually characterized by low-value transactions. Our results, therefore, are robust to the change in the consumption basket that took place during the pandemic.

Finally, in Ardizzi et al. (2019) the authors used LP methods and payment transaction data to evaluate the effect of uncertainty and the fear of fraud on private consumption and the preference for cash proxied by the cash-card ratio. In particular, these authors observe a positive correlation over time between the uncertainty indicators (EPU, fraud rate on electronic payments) and the cash-card ration, as well as a negative correlation over time between the indicators of the business cycle and the cash-card ratio. This last evidence suggests a countercyclical pattern that is consistent with the literature on cash demand showing that the higher the standard of living, the lower the relative use of cash and the greater the demand for alternative payment instruments (i.e. Stix, 2004; Lippi and Secchi 2009). Our analysis differs from their paper in several respects. First, we intend to identify the impact of the pandemic in the recent period and not of a generic uncertainty indicator on a longer perspective. Second, we consider a large number of payment habits indicators, with a special focus on the most innovative instruments. Third, we document an important difference in the estimation results. Our analysis shows that the pandemic - that also raised uncertainty in the economy and worsened the business cycle - led to a decline not an increase in the cash-card ratio. The reason is that payment habits seem to be much more reactive to changes in the relative cost of the various instruments (implicit cost of mobility and fear of infection) rather than to fluctuations in the business cycle.

The remainder of the paper is the following. Section 2 describes the data used in the empirical analysis. Section 3 provides some preliminary results on the impact of the pandemic on payment habits using a standard step-dummy approach while in Section 4 we provide an in-depth assessment of such effects, including their persistence, in a local

\footnotetext{
${ }^{5}$ For the case of Italy, Aprigliano et al. (2019) exploited the forecasting power of monthly payment data for real GDP using a mixed frequency factor model. Recently, Delle Monache et al. (2020) include payment data in a large set of high-frequency data to develop a weekly indicator for real GDP in Italy.
} 
projection framework. In Section 5, we study the effects on banknotes in circulation while in Section 6 we provide some robustness checks and additional evidence from survey data. Finally, in Section 7 we offer some concluding remarks and discuss the related policy implications.

\section{Data and descriptive analysis}

In this section, we describe the source and the construction of the variables we use in the empirical analysis. Overall, we exploit high-frequency data from different sources and construct a unique data set at daily frequency for the Italian economy, covering the period between January 2019 and August 2020. The sample, therefore, includes the period before the outbreak of the pandemic, which is important as it is obviously important to properly account for previously ongoing trends. Moreover, it comprises the various phases of the emergency in Italy and the associated measures to limit the infection, namely the severe lockdown from March to May and its recessionary effects and the subsequent softening of the restrictions to social mobility and the recovery in economic activity.

\subsection{Indicators of the pandemic's intensity}

To obtain a measure of the pandemic intensity, we rely on unconventional data, namely Google Trends, which is an effective way to gauge the popularity of certain terms and topics among a targeted audience in terms of search frequency. Google Trends provides only indicators representing how many web searches people do for particular keywords, not publishing the actual numbers. The indicators are available in the form of time series of whole numbers, normalised so that their maximum equals 100 . Accordingly we construct a single indicator for each day of the sample period resulting from searches in Italian language relating infection to payments, as follows: $i$ ) "come pagare Coronavirus", ii) "pos Coronavirus", iii)"banconote Coronavirus", iv) "contanti Coronavirus" and $v$ ) "Bancomat Coronavirus".

Moreover, we take the official data on "new infections" provided by the media on a daily basis, as these announcements represented the most important news for households and firms about the evolution of the pandemic, and represent an alternative measure of the COVID-19 shock. To compare data to the Google Trends indicator we normalised the series so that the maximum equals 100 .

Figure 1 depicts the daily Google Trend index about the COVID-19 event in comparison to the index of official data on new infections. The two indexes show a similar pattern (the unconditional serial correlation coefficient is 0.6) with some differences. In particular, the Google Trend indicator shows a more volatile distribution (with the most frequent values on March 2020) and captures the episode of the pandemic outbreak in advance with respect to the official data index, which shows a unimodal serial distribution with one clear peak on April (in line with the deterministic trend of infection in Italy). This pattern reflects the view that Google Trend captured the news about the pandemic coming from the rest of the world (e.g. the rise of infection in Asia), as well as some about local epidemic breakouts in Italy (e.g. the first cases of infection in Lombardia and the case of two Chinese tourists in Rome at the end of January). For this reason, the official national statistics do not report such information.

\subsection{Indicators of payment habits}

In this section, we describe the definition and computation of the indicators of payment habits, including their source and treatment of the data. For our analysis, we collect daily time series covering the period between January 2019 and August 2020. 
Survey data suggest a large recourse to cash in Italy for transaction purposes. According to the Eurosystem's 2016 Survey on the use of cash by households (SUCH), Italians pay in cash around 68 percent of purchases at the point-of-sale, 29 percent using cards and 3 percent relying on other payment instruments ${ }^{6}$; moreover, people mostly withdraw cash at ATMs rather than at bank counters (Rocco, 2019; Esselink and Hernandez, 2017). Card-based payment instruments represent the main substitute for cash in retail payment, as well as the main channel for cash provision. Credit cards also represent the most common forms of payment for e-commerce transactions, also in combination with PayPal and the new e-Wallet such as Apple Pay, Google Pay and Samsung Pay. According to the Survey of Household Income and Wealth (SHIW) of the Bank of Italy, conducted in 2016, 76 percent of the households in Italy hold a debit card, versus 30 percent for credit cards and 25 for prepaid cards. Based on this evidence, our analysis mostly relies on transaction data with debit and credit card.

A first block of variables is based on information provided by BI-COMP, which is the retail settlement system managed by the Bank of Italy. This system provides daily data on the value and number of transactions of all interbank payments using several instruments, such as credit transfers, direct debits, debit cards and cheques. On average, it handles around 5-6 million payments per day (which amounts to. around EUR 4-5 billion per year) and covers about 60 percent of the entire payment system. As already mentioned, we focus our attention on Point-of-Sale (POS) transactions and cash withdrawals at automated teller machines (ATM) by means of debit cards.

By contrast, consumers mostly use credit cards for online purchases (e-commerce) or for specific transactions, such as travel expenses and purchases of durable goods. Moreover, credit cards are usually characterized by contact-less technologies. Such kind of data are not settled in the BI-COMP system but are available from private processing entities. Therefore, a second block of payment variables considers the diffusion of ecommerce and contact-less transactions based on ad-hoc elaborations of a representative sample of high-frequency credit card data ( $60 \%$ of total credit card transactions).

Since ATM withdrawals may be a good proxy of the demand for cash for transaction purposes (Carbo-Valverde and Rodriguez-Fernandez, 2014), we use the "cash-card ratio" - the ratio between the daily value of ATM cash withdrawals and the daily value of POS card operations - as a measure of the preference for cash against electronic payments (see Ardizzi and Iachini 2013). If ATM transactions tend to grow more than POS transactions, the indicator increases and signals an increasing preference of consumers for cash. Notice that BI-COMP settles only the so-called "not-on-us" ATM transactions, namely operations implying an additional fee for the consumer where the issuing bank of the card is different from the ATM bank. In this case, an increase in the cash-card ratio better captures a higher attitude for cash, as consumers are willing to pay a fee. The opposite story holds when the cash-card ratio declines, with people exhibiting a preference for electronic or digital transactions. BI-COMP figures are strongly correlated with the statistics on total debit card transactions (for both ATM and POS), available at quarterly frequency from the Supervisory Reports to the Bank of Italy ${ }^{7}$.

\footnotetext{
${ }^{6}$ Regarding the number of transactions, people made around 86 per cent of payments in cash, 13 per cent with cards and 1 per cent with other payment instruments. Consumers mainly used cash for low-value payments, as opposed to other instruments for high-value transactions. We notice that SUCH focuses only on transactions at POS, where the value of payments is typically low.

7. The simple cross-correlation index (Pearson) between the BI-COMP cash card ratio and the cash card ratio based on the reports of the whole banking system during the period 2017.Q1-2020.Q2 is over 0.8.
} 
We also compute the cash-card ratio as the ratio of the number of transactions at ATM to the number of transactions at POS. A visual inspection since the first official cases of infection in Italy (i.e. February 21th) shows that both ATM withdrawals and POS transactions dramatically collapsed, reflecting the negative effects of the lockdown measures on social mobility and, in turn, on private consumption (Figure 2). The decline in the cash-card ratio (see Figure 3) would suggest a higher preference for payment at POS rather than using cash during the pandemic. However, a downward trend of the cash-card ratio was already in place since the last quarter of 2019, maybe reflecting the announcement effect of the last "Fiscal Decree" 8 in Italy (approved on December 2019), aimed at encouraging the use of electronic payments at the point of sales.

An alternative indicator of payment habits is the average value of transactions at POS. The economic reason, under normal conditions, is that a declining trend in this indicator should capture a more and more intense use of cards at POS for low-value transactions instead of cash, thus suggesting an increasing preference for electronic payment for transaction purposes. This interpretation is sharply at odds with evidence in Figure $3 b$ where the average value at POS increases during the pandemic, when the restrictions to mobility led consumers to lower the frequency of purchases at shops while increasing the amount spent.

We also consider the average value of withdrawals at ATM, which is central in inventory models of money demand, as consumers make withdrawals also to hoard cash. While a clear-cut separation between cash withdrawals for transaction or for precautionary motives is not straightforward, we argue that during the pandemic an increase in this variable is likely to capture several factors, such as the provisioning of liquidity for future purchases in the face of restrictions. Moreover, the fear of infection and the substantial difficulty to go physically to bank branches during the lockdown could have implied an impairment on the cash cycle and accelerate the on-going substitution effect from cash withdrawals at the bank counters - usually characterized by large amounts - to ATM withdrawals. In Section 5, we give an in-depth assessment of the effects of the pandemic on cash demand by looking at banknotes in circulation.

We also consider indicators capturing the preference for the most innovative payment instruments. The pandemic, indeed, forced banks, Fintech companies and retailers to roll out digital and non-touch payment mechanisms. ${ }^{9}$ We focus on contactless cards that transmit payment messages to a POS device with or without the need to insert, swipe or to key in the Personal Identification Number (i.e. the PIN code). For contact-less transactions, when the transaction exceeds a certain limit (i.e. 25 euro), the merchant still requests the PIN code or the signature. Following the statement of the EBA (March 2020) allowing payment service provider to increase the limit for no-PIN contactless transaction from 25 to 50 euro, in several countries (including Italy), card schemes and banks in cooperation with the retailers have been responding to pandemic fears by planning to raise as soon as possible the limits on their contactless card transactions. In our analysis, we consider the share of contact-less card transactions as a proxy of innovation in payment habits.

\footnotetext{
${ }^{8}$ See Law Decree n. 124/2019, which entered into force on July 2020 for electronic payment provisions.

${ }^{9}$ The contactless payment systems use radio-frequency identification or near field communication for making secure payments and can involve credit and debit cards, key fobs, smart cards, smartphones and wearable devices. The embedded integrated circuit chip and antenna enable consumers to wave their card, fob, or handheld device over a reader at the POS terminal. People make contact-less payments in close physical proximity. Mobile payments, instead, use broad-area cellular or Wi-Fi networks and do not involve close physical proximity.
} 
Figure 4 shows that the diffusion of the pandemic was associated to a more intensive use of such innovative payment means. The propensity to pay in contactless modality at POS, already characterized by an upward trend, further increased to 50\% since the first week of March (it was 30\% in the previous year) without any sign of reverse in this trend. In the same vein, the share of online transactions reached a peak of $40 \%$ in March and April, while somewhat declining in the following months to values still higher with respect to one year before.

\section{A primer on the effects of the pandemic on payment habits}

In this Section, we evaluate the recent trends of the main payment habits indicators (i.e. POS and ATM average value of transactions, cash-card ratio expressed in terms of both values and volumes, the share of e-commerce and contactless card transactions). During the pandemic crisis, such indicators show a significant level shift with respect to the previous period, that we can evaluate thorough a standard regression analysis with step-dummy variables. Hence, we run regression models where the dependent variables are the payment habit indicators, and the regressors are binary (i.e. 0-1) dummy variables equal to one for specific periods during the pandemic. More specifically, we consider a dummy variable equal to one for the days in the period March $8^{\text {th }}$ - May $3^{\text {st }}$, denoted as "lockdown". For the lockdown period, we consider the date of entry into force and the period of validity of the Italian Decree of the President of the Council of Ministers, which suspended several business activities to contain the infection throughout the national territory and imposed dramatic restrictions to social mobility. Moreover, we define a second dummy variable equal to one for the days after $3^{\text {st }}$ May, denoted as "post-lockdown", which is useful to investigate the average response of the payment indicators during a progressive easing of the restrictive measures. After the lockdown, the perception of the infection risk could have remained relevant among people. Hence, consumers' behavior were likely to be affected by the pandemic, notwithstanding the re-opening of all commercial and recreation activities. Accordingly, we estimate a battery of equations of the following form:

$y_{t}=\alpha+\beta \cdot$ lockdown $+\gamma \cdot$ post-lockdown $+\mu_{t}$

The logic of this empirical analysis is that the dummy variables capture a kind of deterministic "treatment effect" on outcome variables induced by the outbreak of the pandemic. In other words, we are testing if consumer payment habits would have remained broadly stable to pre-pandemic period in the absence of such treatment. Our hypothesis is that differences observed in the data can be mainly attributed to the COVID-19 crisis. In this regard, the coefficient $\beta$ captures the average affect during the lockdown, while $\gamma$ measures the impact in the subsequent period. If $\beta$ and $\gamma$ exhibit statistically significant values with the same sign, we have a rough indication of a persistent effect on payment habits even if with a different magnitude. The specification also includes working daily and weekly dummies to detect seasonality. We base inference on robust standard errors for both heteroskedasticity and autocorrelation in the residuals. Table 1 summarizes the estimation results for the outcome variables.

Models (1) and (2) show the response of the cash-card ratio measures, which decreased, on average, both during and following the lockdown. The decline is about 10 and 5 basis points, respectively, for values and volume of transactions. We also find that both POS and, especially, ATM average transaction value increased after the lockdown, as suggested by Models (3)-(4). In particular, the ATM average value increased by about 16 percent during the lockdown and more than 7 percent in the following period. POS average value increased too, but at lower rates. These results corroborate the view that 
social mobility restrictions during the emergency reduced the possibilities to make frequent (and low value) operations at the physical premises and increased cash-in-hand for precautionary reasons.

As regards the payment technology adoption, results in Models (5) and (6) indicate that both the percentage of e-commerce and contact-less transactions increased - on average - during the emergency (both by about 20 basis points); for the contact-less card usage, such increase remains high also after the lockdown. Overall, these results show that the pandemic led to a significant break in the mean of the outcome variables, which only partially reversed following the easing of the lockdown measures. As a result, consumer payments have probably shifted into a "new-normal" characterized by a larger amount of electronic payments and a lower preference for cash at the point-ofsale. We provide more details about the causal nexus between payment habits and the outbreak of the COVID-19 into an impulse-propagation paradigm in Section 4.

Table 1 - Effects of the pandemic on payment habits: stylized facts

\begin{tabular}{lcccccc}
\hline & $\begin{array}{c}\text { cash-card } \\
\text { (values) }\end{array}$ & $\begin{array}{c}\text { cash-card } \\
\text { (volumes })\end{array}$ & $\begin{array}{c}\text { POS } \\
\text { avg.value }\end{array}$ & $\begin{array}{c}\text { ATM } \\
\text { avg.value }\end{array}$ & $\begin{array}{c}\text { fraction of } \\
\text { e-commerce }\end{array}$ & $\begin{array}{c}\text { fraction of } \\
\text { contactless }\end{array}$ \\
\cline { 2 - 7 } & $(1)$ & $(2)$ & $(3)$ & $(4)$ & $(5)$ & $(6)$ \\
\hline \hline lockdown & $-0.097 * * *$ & $-0.049 * * *$ & $0.076 * * *$ & $0.156 * * *$ & $0.187 * * *$ & $0.201 * *$ \\
& $(0.007)$ & $(0.003)$ & $(0.009)$ & $(0.008)$ & $(0.019)$ & $(0.001)$ \\
post-lockdown & $-0.116 * * *$ & $-0.051 * * *$ & $0.025 * * *$ & $0.074 * * *$ & $0.039 * * *$ & $0.245 * * *$ \\
& $(0.004)$ & $(0.001)$ & $(0.004)$ & $(0.004)$ & $(0.006)$ & $(0.004)$ \\
\hline Observations & 420 & 420 & 420 & 420 & 420 & 420 \\
Adj. R-squared & 0.699 & 0.757 & 0.657 & 0.823 & 0.845 & 0.686 \\
\hline \hline
\end{tabular}

Notes: lockdown is a binary variable equal to one for the days between March $8^{\text {th }} 2020$ and May $3^{\text {st }} 2020$; post-lockdown is a binary variable equal to one for the days after May $3^{\text {st }} 2020$. Estimated coefficients based on standard OLS regressions on daily data. The specification includes working daily and weekly dummies to detect seasonality. Robust standard errors in parentheses. $* * *, * * *$ denote statistical significance at $1 \%$, $5 \%$ and $10 \%$, respectively.

\section{Impulse response analysis on payment habits}

\subsection{Methodology}

We evaluate the dynamic effects of the COVID-19 shock on the outcome variables by using the local projection method proposed by Jordà (2005) and recently adopted in several macroeconomic studies. Accordingly, we obtain the Impulse Response Functions (IRFs) of variable $y$ at forecast horizon $h$ to the exogenous measures related to COVID-19 $(x)$ running the following set of regressions with variables in levels:

$y_{t+h}=\alpha_{h}+\sum_{i=1}^{q} \beta_{i, h} y_{t-i}+\gamma_{h} x_{t}+\varepsilon_{t+h}$

where each forecast horizon $h$ is associated with a different regression, $\alpha_{h}, \beta_{i, h}, \gamma_{h}$ are parameters that may vary across regressions $h$. This is essentially the "local projection" method of estimating impulse response functions, with a potential structure of lags for the endogenous variable $y$ on the right-hand side. However, we find that in the majority of the cases additional lags are not necessary in the specification. Jordà (2005) showed that the error terms arising from these projections are moving-average processes of order $h$ if the specification also includes lags of the dependent variable as additional regressors, thus inducing autocorrelation in the residuals. Therefore, we adopt a GMM estimator based on a heteroskedasticity- and autocorrelation-consistent (HAC) weighting matrix where the specified kernel and the lag order are selected using the 
Newey-West optimal lag-selection algorithm. Finally, when the dependent variable is highly persistent, we rely on the following specifications in first-differences:

$y_{t+h}-y_{t-1}=\alpha_{h}+\sum_{i=1}^{q} \beta_{i, h} \Delta y_{t-i}+\gamma_{h} x_{t}+\varepsilon_{t+h}$

where $\Delta$ denotes the difference operator applied to the outcome variables.

This methodological framework has several advantages. First, local projection is pointwise more robust to model misspecification than impulse responses obtained by Vector Autoregression (VAR) models due to the direct estimation approach versus the VAR iterated simulation procedure. The iterated method, indeed, compounds the errors due to misspecification as a function of the forecast horizon. Second, the local projection estimator can be easily adapted to a nonlinear framework and can easily embed in the statistical model crucial features, such as state, size and sign dependency of the effects.

It is important to remark that having an exogenous factor, we avoid estimating structural local projection, which is not necessarily better than relying on other tools, namely Structural VAR models (SVARs). The conventional wisdom in the empirical literature is that SVARs are more efficient, while LPs are more robust to model misspecification, albeit these remarks are not based on formal analysis and call for further research (see Kilian and Lütkepohl, 2017; Ramey, 2016; Nakamura and Steinsson, 2018; Stock and Watson, 2018). ${ }^{10}$

The specification of the predictive regressions also includes additional variables to deal with the strong seasonality and the calendar effects characterizing the outcome variables. In normal times, the largest amount of payments at POS occur during the weekend, when households have more time to go shopping. Within a month, POS transactions are also more frequent in the third week, in concomitance with the payment of wages and salaries. At the yearly frequency, such payments are particularly large in January, during Easter time (March and April) during summer holidays (June and July) and Christmas time (December). Similar considerations apply to ATM withdrawals. Notice that the extracted data from BI-COMP are available only for working days (i.e. 5 days a week). Therefore, transactions made during weekends and holidays are recorded in the system on Mondays or on the first working day of the week following a holiday. This implies that we deal with large spikes in the data at the beginning of each week. This problem largely attenuates in our empirical analysis since we build the majority of our indicators of payment habits as ratio of variables. ${ }^{11}$ In any event, we include an appropriate set of dummy variables capturing working daily, weekly and monthly seasonal effects. ${ }^{12}$

\footnotetext{
${ }^{10}$ Existing studies provide some useful guidance, but differences in implementation details have led to mixed conclusions (Kilian and Kim, 2011; Nakamura and Steinsson, 2018; Choi and Chudik, 2019). In a very recent contribution, Plagborg-Møller and Wolf (2020) prove that LPs and VARs estimate the same impulse responses in population. In finite samples, VARs that control for a large number of lags will tend to agree at short and medium-long horizons with local projections that also control for a rich set of lags. At longer horizons, the two methods instead may disagree substantially and a bias-variance trade-off can arise.

${ }^{11}$ In order to reduce the volatility of the original series over the weekends, we could take the daily data recorded on Monday and split them with equal weights over Saturday, Sunday and Monday. In this way, we would impute a value also for Saturdays and Sundays. This, however, will lead to daily time series exhibiting "steps" over time and not a continuous time dynamics.

${ }^{12}$ We could apply more sophisticated techniques, such as the seasonal adjustment procedure TBATS, that have been recently proposed in the literature to treat time series with multiple seasonalities (De Livera et al. 2011) and already adopted in Ardizzi et al. (2019) for payment system data. This methodology,
} 


\subsection{Empirical results}

Figure 5 plots the results of the predictive regressions for the outcome variables, with forecast horizon $h$ on the horizontal axis and the estimated coefficients on the vertical axis. The solid lines plot the point estimates while the dotted lines plot the robust confidence bands around those point estimates. The red line refers to impulse response functions to the pandemic intensity, as captured by a Google Trend indicator. The blue line refers to impulse response functions to changes in the time series of official new COVID-19 cases.

We explore the estimated effects over forecast horizons up to five months. In the case of BI-COMP variables, this choice implies computing local projections up to 100 working days. In the case of outcome variables provided by card processors - that are available for all calendar days - we report the effects up to 150 days. Focusing on a long forecast horizon is particularly appropriate, since allows us to evaluate the persistence of the effects during different phases of the epidemiological context and the associated restrictions to limit the infection. As already discussed in the previous Section, the first period refers to most acute phase of the pandemic characterized by the lockdown, the dramatic restrictions to social mobility and the closure of non-essential activities. The estimated impulse responses at horizons $1 \leq h \leq 50$ for BI-COMP variables (working days) and $1 \leq h \leq 70$ for technology indicators (calendar days) mostly detect the effects during the lockdown. The impulse responses at longer forecast horizons (i.e. $h \geq 50$ for BICOMP variables and $h \geq 70$ for technology indicators) instead evaluate the effects in the second period, the one between May and August 2020, characterized by a progressive easing of the lockdown measures and first signs of recovery in economic activity.

By looking at the effects of the pandemic, as captured by the official new cases of infected people, the top-left chart in Figure 5 suggests that, following a 10 percent increase in this indicator, the cash-card ratio declines on impact by about 80 basis points, with the fall being very persistent over time. The point estimates do not show sign of diminishing and the standard errors suggest that this effect remains statistically significant at all forecast horizons. We find a similar pattern using the number of transactions when computing the cash-card ratio, albeit the effects are about one-half in magnitude and slightly less persistent.

The evidence of a negative impact on the cash-card ratio after the lockdown, confirms the preference for cards with respect to cash also in the most recent months. We give more details about the economic reasons behind these patterns by looking at the responses of the individual variables used in the computation of the cash-card ratio (see Figure A1 in Appendix A). Following a large shock hitting the economy, private consumption recorded a dramatic fall. However, the decline in both values and volumes of transactions at POS was much more muted than the decline in ATM withdrawals. A possible explanation is that the fear of infection induced the people to limit the usage of cash and the recourse of ATMs that used by other persons and more exposed to the virus transmission than their own cards.

At the same time, the outbreak of the pandemic significantly raised the average value of transactions at POS. The figure, however, suggests a hump-shaped response. The magnitude of the increase is about 0.1 percent on impact but becomes larger reaching a maximum (more than 0.2 percent) after one month. Then, the estimated effect tends to decline after the lockdown, with indications of absorption at the end of the

however, would require much longer time series. The quality of the data for some time series prevented us to use such procedure. 
sample period. The raise in the average value at POS could reflect the fact that households went shopping less frequently during the period of restrictions to mobility and, therefore, made larger payments. Following the outbreak of the pandemic, indeed, the response of the number of transactions was stronger than the values of transactions (see again Figure A1), especially during the lockdown period.

An interesting result is that the average withdrawal at ATMs increased as well in reaction to the pandemic. The increase was large and very persistent. The estimated coefficients show a rise on impact by about 0.4 percent, followed by a slight decline after the lockdown. While partly reversing, this effect remains statistically significant for the entire forecast period. At longer horizons, it still stood at values around 0.1 percent. People picked cash up less frequently during the pandemic but withdrew a larger amount of money reflecting a higher demand for precautionary motives induced by restrictions to mobility.

The bottom part of Figure 5 shows the response of the means of payment more related to technological innovation. The news on the pandemic immediately raised the fraction of e-commerce transactions by about 40 basis points. This effect is persistent for about two months, while starting to decline afterwards, when the magnitude of the impact progressively reduces coming back to the pre-pandemic values. Figure A2 shows that during the lockdown period e-commerce transactions fell by about 2 percent in March and April while suddenly recovering afterward. The collapse in the other cardbased transactions was much stronger (about 20 percent) and exhibited a delayed recovery with respect to e-commerce transactions. This result suggests that technology and e-commerce helped sustaining the economy in the face of an unprecedented shock hitting the economy.

Another important result is the positive response of the fraction of contactless card transactions. This variable increased on impact by about 80 basis points. The point estimates and the associated standard errors suggest that this effect is highly statistically significant and very persistent over the entire forecast horizon. After the lockdown, the point estimates slightly decline reaching 60 basis points at the end of the forecast period. Figure A2, again, shows that the technology adoption is crucial for economic growth. During the lockdown and the associated recession, contactless transactions declined by about 5 percent against a fall by about 35 percent for non-contactless transactions. When the economy started to improve and non-essential activities re-opened, the effect on contactless transactions turned rapidly on a positive territory, suggesting an expansion by about 10 percent over the last months. On the contrary, the effect on non-contactless transactions remains negative over the forecast horizon, albeit attenuating. In the recent months, it still suggests a fall by about 10 percent.

By using the Google Trend indicator as a measure of the perception of risk associated to the pandemic, the main picture remains broadly unchanged. We find smaller effects in magnitude - albeit highly statistically significant - for the BI-COMP variables (i.e. cash-card ratio and average value of both ATM and POS transactions) at shorter horizons. In the other cases, notably the indicators related to innovative payment instruments, the effects are very similar and not statistically different one another.

The difference in the estimation results can reflect the relatively larger amount of noise in the Google Trend indicator, which can make it more difficult to detect the economic effects in some cases. An alternative explanation is that this indicator is able to isolate one channel of transmission of the pandemic shock on payment habits, namely the different fear of infection when using different means of payment. The official new cases of infected people, indeed, can also capture important re-composition effects on 
households' consumption basket related to the lockdown measures. The closure of nonessential activities, such as recreation ones, restaurants and a large number of shops induced a large shift of households' purchases towards certain goods in specific shops, such as foods at supermarkets and pharmaceutical products. We speculate that the use of unconventional data may help to identify the "implicit" cost of the fear of infection using different payment instruments.

\section{Effects of the pandemic on the circulation of banknotes}

In this section, we give an assessment of the effects of the pandemic on alternative cash related variables, namely the value of banknotes in circulation and its components. The main aim is to corroborate previous evidence of a diminishing role of cash for transaction purposes against a potential role played by the precautionary demand. To this end, we use the daily information provided by branches of the Bank of Italy regarding the net issuance, which is equal to the difference between the total value of banknotes issued by the Bank of Italy (withdrawals) in a given period and the total value of banknotes returned to the Bank in the same date (lodgements). This measure permits to capture the impact on cash demand of domestic-specific shocks, albeit including banknotes issued in Italy and sent abroad as well as banknotes issued abroad that have entered the country. ${ }^{13}$ For the purpose of our analysis, we also take on board a breakdown of total circulation by denomination. Accordingly, we consider lowdenomination banknotes (i.e. we sum up $€ 5$ and $€ 10$ notes) with respect to medium- (i.e. we sum up $€ 20$ and $€ 50$ notes) and high-denomination ones (i.e. we sum up $€ 100, € 200$, and $€ 500$ notes). It is important to remark that withdrawals at ATMs involved mainly medium-denomination notes (i.e. $€ 20$ and $€ 50$ notes), which are used both for immediate transaction and precautionary purposes.

Figure 6 shows an upward trend in total circulation following the outbreak of the pandemic, which seems to be at odds with our previous evidence of cash substitution in the economy. However, this broad picture masks substantial heterogeneity across the main components of circulation, which also respond to a number of confounding factors. First, in order to corroborate the evidence of cash substitution with other payment instruments it is important to distinguish cash demand held for transaction purposes which is likely to be proxied by low-denomination notes - from cash demand for precautionary reasons - which may reflect holdings of medium- and high-denomination notes. This is relevant as the pandemic raised uncertainty in the economy and induced restrictions to mobility, with people retaining cash for future purchases.

Second, looking at the past, the evidence of negative values for the cumulative net issuance of some denomination notes (i.e. $€ 10, € 200$ and $€ 500$ ) relates to the fact that Italy is structurally affected by net inflows from abroad, at least in part connected with tourism, with negative and persistent effects on banknotes in circulation. The outbreak of the pandemic could have dramatically changed this paradigm. The key mechanism at work is that the sudden and strong decline in tourism and flows of people coming from abroad for business could have led to a strong reduction in the value of

\footnotetext{
${ }^{13}$ Circulation is calculated as the cumulated net issuance from January 2002. It is also possible to refer to an alternative measure for the circulation of cash (usually used in each euro-area country), computed on the liability side of the National Central Bank (NCB) balance sheets. This measure is based on a conventional criterion that attributes to each country a share in the euro-area total issues relative to the respective share in the ECB's capital key. The capital key is determined on annual basis on the population and GDP of the respective country. Differently from the net issuance, this measure does not permit to capture the impact on circulation of domestic-specific shocks, as its dynamics can reflect shocks that are specific to the euro area as a whole.
} 
banknotes returned to the central bank, which, in turn, generates an immediate and "mechanic" increase in circulation. To improve the identification of the effects of the pandemic on usage of banknotes by residents in Italy, we consider in the empirical analysis an additional control variable for cash flows from abroad. Accordingly, we rely on unconventional data on mobile network for foreign travellers described in Carboni et al. (2020) as a "rough" daily proxy for cash flows from abroad. During the sanitary emergency, this variable helped estimating tourism expenditure in the balance of payments and temporary replaced the monthly information usually recorded by the central bank in an ad-hoc survey.

Third, the pandemic represented an unprecedented shock hitting the cash cycle, which calls for assessing the response of withdrawals and lodgements, separately. In the data, indeed, we observe an exceptional decline of lodgement of banknotes to the Bank of Italy, which could be related to the closure of non-essential business activities during the lockdown and difficulties for people to deposit money at the bank tellers. Moreover, households and businesses may have decided to retain cash for precautionary reasons, given the dramatic recession hitting the economy and its implications for future employment and disposable income. At the same time, it was recorded a decline in withdrawals from the central bank but much less severe than for lodgements, as they are needed for cash payments for some recurrent items such as cash pensions payments at the Postal Offices.

We formally test for a significant upward shift in banknotes in circulation and its main components during the pandemic by running the same regressions with dummy variables described in Section 3. As the stock of banknotes in circulation exhibits strong non-stationary, we deal with all dependent variables in first-differences. We report the estimated coefficients in Table 2.

Table 2 - Effects of the pandemic on banknotes in circulation: stylized facts

\begin{tabular}{lc|cc|ccc}
\hline & circulation & lodgments & withdrawals & low-denom & med-denom & high-denom \\
& $(1)$ & $(2)$ & $(3)$ & $(4)$ & $(5)$ & $(6)$ \\
\hline \hline lockdown & $0.085 * *$ & $-0.858 * * *$ & -0.212 & $-0.157 * *$ & $0.051 * *$ & $0.024 * * *$ \\
& $(0.037)$ & $(0.238)$ & $(0.281)$ & $(0.078)$ & $(0.025)$ & $(0.007)$ \\
post-lockdown & $0.068 * * *$ & $-0.392 * * *$ & 0.135 & -0.011 & $0.040 * * *$ & $0.018 * * *$ \\
& $(0.012)$ & $(0.054)$ & $(0.083)$ & $(0.064)$ & $(0.013)$ & $(0.004)$ \\
foreign flows & -0.010 & $0.182 * * *$ & 0.070 & $-0.095 * *$ & -0.002 & $-0.014 * * *$ \\
& $(0.017)$ & $(0.057)$ & $(0.075)$ & $(0.039)$ & $(0.011)$ & $(0.003)$ \\
\hline Observations & 422 & 422 & 422 & 422 & 422 & 422 \\
Adj. R-squared & 0.225 & 0.242 & 0.136 & 0.089 & 0.212 & 0.338 \\
\hline
\end{tabular}

Notes: the dependent variables are the following: circulation = change in total value of banknotes in circulation; lodgments $=$ log-level of lodgments to the central bank; withdrawals $=\log$-level of withdrawals from the central bank; low-denom = change in the value of low-denomination notes; med_denom $=$ change in the value of medium-denomination notes; high_denom $=$ change in the value of high-denomination notes; foreign flows $=$ logarithm of the number of travelers recorded in the mobile network. lockdown is a binary variable equal to one for the days between March $8^{\text {th }} 2020$ and May $3^{\text {st }}$ 2020. Post-lockdown is a binary variable equal to one for the days after May $3^{\text {st }} 2020$. Estimated coefficients based on standard OLS regressions on daily data. The specification includes the constant term, working daily and weekly dummies to detect seasonality. Robust standard errors in parentheses. $* * *, * *, *$ denote statistical significance at $1 \%, 5 \%$ and $10 \%$, respectively.

The results suggest that total circulation negatively and significantly correlates to our proxy variable for cash from abroad, especially for lodgements, low- and highdenomination notes. Interestingly, the negative effect of the pandemic is significant and 
persistent for lodgements to the central bank, with flows of banknotes reducing by about 90 and 40 percent, respectively, during and after the lockdown. On the contrary, we find no significant effect on withdrawals. Net of the foreign component, flows of lowdenomination notes significantly decreased during the lockdown, consistently with a weaker dynamics of the transaction component. Flows of both medium- and highdenomination notes instead markedly increased both during and after the lockdown. ${ }^{14}$ The effect is stronger for medium-denomination notes (50 and 40 basis points during and after the lockdown, respectively) than for large-denomination ones (about 20 basis points in both periods).

In Figure 7, we present the estimated local projections at forecast horizons up to five months. Total net issuance increased persistently during the pandemic but flows from abroad significantly matter in explaining these developments. Net of this foreign factor, the impulse responses become not statistically significant at all forecast horizons. It is interesting to look at the response of total withdrawals and lodgements, separately (see Figures A3-A4 in Appendix A). We find a pronounced and significant reduction of banknotes lodged at the Bank of Italy, as opposed to a negligible effect in the case of withdrawals. The decline of lodgement of banknotes, only partially explained by the reduction of foreign inflows, could be due to the closure of non-essential activities during the lockdown. Moreover, households and businesses may have decided to hold cash for precautionary reasons instead of depositing banknotes into the banking system. This effect remains significant even after the lockdown. On the other hand, the impact of the pandemic on withdrawals is much smaller in magnitude, confined to the lockdown period and characterized by wider confidence bands.

As for the impulse responses associated to the different denomination notes, we focus our attention on results net of our proxy for cash flows from abroad (see again Figure 7). We find a negative and significant effect on low-denomination banknotes, which decline by about 2 percent at forecast horizons up to two months. After the lockdown, the effect becomes negligible consistently with the recovery in economic activity. The estimated effect on medium-denomination notes is instead positive but characterized by wide confidence bands, maybe reflecting the hybrid nature of such notes, usually adopted by consumers for both transaction and precautionary reasons. Finally, we find a positive and persistent effect on high-denomination notes, albeit quite small in magnitude.

Overall, these results corroborate the view of a decline in the usage of cash when making transactions but also remark the potential role played by the precautionary motive in the recent dynamics of circulation, similarly to what found for the average withdrawal at ATM. A proper quantification, however, remains difficult since part of the precautionary component of cash demand may reflect the "involuntary" effect on cash balances stemming from the impairment in the cash cycle.

\section{Robustness checks and additional evidence}

In this Section, we test the robustness of our main results along several dimensions. We report the figures stemming from this additional analysis in Appendix B.

\footnotetext{
${ }^{14} \mathrm{An}$ important caveat in the interpretation of these results is that banknotes in circulation can also embed transactions within the underground or illegal economies, which is likely to fuel into the precautionary demand for cash. Unfortunately, in our framework we are not able to disentangle such phenomena. Evidence for Italy suggests that criminality and tax evasion are positively correlated to the use of cash but their correlations with the observed discontinuity among provinces are not predominant with respect to innovation and financial literacy (Ardizzi et al., 2020).
} 
First, we address the role of the sample period. Local projections are based on "expanded" regressions, as the sample size declines with the forecast horizon. To test the robustness of our estimates we also evaluate the effects stemming from "rolling" regressions, where the sample size remains constant for all horizons. This methodology is useful to assess the stability of the model parameters with respect to time. Results remain broadly unchanged, albeit in some cases the difference between estimates based on Google Trend indicator and the time series of official infections become larger.

Second, the effect of the pandemic on consumption has been quite heterogeneous across categories of expenditure, especially during the lockdown period. Both national statistics and evidence for other countries (see Carvalho et al., 2020) show an increase in purchases related to basic necessities, such as foodstuffs and healthcare and pharmacy products, characterized by very low demand elasticity. In contrast, expenditure on goods and services related to recreation activities and personal services has all but vanished. This gives rise to a large reallocation in the consumption basket, which can represents a confounding factor affecting our main results. We evaluate the substitution effects in favor of the contactless technology by focusing on transactions that are specific to the grocery sector, which has been more resilient during the emergency and less affected by composition effects. The results suggest that the effect is even stronger than that recorded for the whole economy. This result is important because purchases in the grocery sector are usually characterized by small-value payments. This experiment cannot be carried out for the other payment habit indicators for the lack of data.

As additional evidence of demand-side effects, we now discuss the consumers' opinions. In July 2020, ECB conducted a survey in all euro area countries to measure the impact of the pandemic on cash trends and payment behaviors (IMPACT survey). ${ }^{15}$ Results for Italy show that more than half of the respondents reported using cards and cash as they did before the start of the pandemic. However, 32 percent of interviewed declared to use less often cash than before, while 27 percent used more often cards. Contactless payments were used more often by 31 percent of them.

People using less cash were also asked to indicate - from a given list - reasons for avoiding the use of cash in physical places since the pandemic started. The most mentioned reason was the fact that paying electronically had been made more convenient (48 percent of respondents), likely due to the possibilities to pay contactless. The risk of being infected by the virus was another main driver, via banknotes for 27 percent of interviewed or via hand contact or the proximity to the cashier for 21 percent. Another important reason was that the government recommended paying cashless as much as possible (26 percent). The access to cash was not a major issue, since only 12 percent of respondents reported they could not withdraw cash as easily as before.

Looking forward, about 60 percent of the respondents who reported using less cash declared that they would certainly continue to pay less with cash and about 34 percent probably will continue to use less cash, thus confirming a permanent change in payment behaviors even when the crisis will be over.

\section{Concluding remarks}

In this paper, we investigate the dynamic effects of the spread of COVID-19 on a number of high-frequency indicators of payment habits and cash demand in Italy. We evaluate such effects by using the local projection method by Jordà (2005), adopted in

\footnotetext{
${ }^{15}$ The results of the IMPACT survey for euro area are included in the ECB report on the Study on the payment attitudes of consumers in the euro area (SPACE) published in December 2020 (ECB 2020).
} 
several macroeconomic studies. We measure the intensity of the pandemic by relying on unconventional data, namely Google Trends, which is an effective way to gauge the popularity of certain terms and topics among a targeted audience in terms of search frequency. Moreover, we consider the official daily data on "new infections", as these data were, by far, the most important news for households and firms about the evolution of the pandemic. As regards the indicators of payment habits and cash usage, we rely on data collected at the Bank of Italy through relevant settlement and processing systems, as well as information provided by local branches regarding net issuances for different denominations, with the aim to catch the transaction and precautionary motive of cash holding in the economy.

According to our estimation results, the sanitary emergency and the government measures to contain the diffusion of infection have intensified electronic payments at the point of sale with respect to cash withdrawals, notably by means of contactless cards and e-commerce options. These developments sustained private consumption during the lockdown and the severe recession hitting the economy. Interestingly, these effects seems to be persistent and are still going-on notwithstanding the removal of the lockdown measures. The pandemic, therefore, could represent a structural break in consumer payment habits and a "game-changer" in the process of substitution of the various means of payment in the society.

Digital payment services have therefore proved to be a fundamental resource for both firms and consumers, modifying traditional exchange processes and allowing greater continuity of their daily activities. Overall, our results suggest that policies aiming at accelerating the digital transformation of the economy and innovation in payment habits can potentially make the economy more resilient to adverse shock and more competitive in terms of consumer and business opportunities. In this paper, we do not consider all forms of digital payments, such as mobile and e-payment solutions because of the lack of data. Looking forward, we are planning to build up an extensive database with transaction data for more innovative cashless instruments, also in order to enrich our empirical analysis.

As regard the effect on cash balances, we provide evidence of a rise of banknotes in circulation and an increase in the average transaction value of ATM cash withdrawals. These figures relate to the impairment in the cash cycle during the lockdown, especially regarding the collapse of lodgements to the central bank, but also call for a potential role played by the precautionary motive in a period of a worsening of the economic outlook. While a proper quantification of such factors remains challenging and left for future research, we argue that ensuring an adequate availability of cash for households and businesses is crucial for a social and economic perspective. 


\section{References}

Alvarez, F.E., Lippi, F. (2009). Financial innovation and the transactions demand for cash. Econometrica, 77, 363-402.

Alvarez, F.E., Lippi, F. (2017). Cash burns: An inventory model with a cash-credit choice. Journal of Monetary Economics, 90, 99-112.

Arango, C., Huynh, K.P., Sabetti, L. (2015). Consumer Payment Choice: Merchant Card Acceptance versus Pricing Incentives. Journal of Banking and Finance, 55, 130-141

Aprigliano, V., Ardizzi, G., Monteforte, L. (2019). Using the payment system data to forecast economic activity. International Journal of Central Banking, 15, 55-80.

Ardizzi, G., Bonifacio, E., Demma, C., Painelli, L. (2020). Regional Differences in Retail Payment Habits in Italy. Bank of Italy, Occasional Papers, forthcoming.

Ardizzi, G., Emiliozzi, S., Marcucci, J., Monteforte, L. (2019). News and consumer card payments. Bank of Italy, Economic Working Papers n. 1233.

Ardizzi, G., Iachini, E. (2013). Why are payment habits so heterogeneous across and within countries? Evidence from European countries and Italian regions. Bank of Italy, Occasional Papers n. 144.

Ardizzi, G., Savini Zangrandi, M. (2018). The Impact of the Interchange Fee Regulation on Merchants: Evidence from Italy. Bank of Italy, Occasional Paper n. 434.

Auer, R., Corneli, G. and Frost, J. (2020). COVID-19, cash, and the future of payments. BIS Bulletin n. 3, April 2020.

Bagnall, J., Bounie, D., Huynh, K.P., Kosse, A., Schmidt, T., Schuh, S. and Stix, H. (2016). Consumer cash usage: a cross-country comparison with payment diary survey data. International Journal of Central Banking, 12, 1-61.

Bank of Italy (2012). The social costs of payment instruments in Italy. Bank of Italy, Institutional Issues, December 2012.

Bank of Italy (2020). Il costo sociale degli strumenti di pagamento in Italia - I risultati della seconda indagine. Bank of Italy, Institutional Issues, March 2020.

Bech M., Faruqui U., Ougaard F., Picillo C. (2018). Payments are a-changing but cash still rules. Bank for International Settlement Quarterly Review, pp. 67-80.

Borzekowski, R., E. Kiser, A. Shaista. (2008). Consumers' Use of Debit Cards: Patterns, Preferences, and Price Response. Journal of Money, Credit and Banking, 40, 149-72.

Bounie, D., Camara, Y., Galbraith, J.W. (2020). Consumers' Mobility, Expenditure and Online-Offline Substitution Response to COVID-19: Evidence from French Transaction Data. Institut Polytechnique de Paris, McGill University, manuscript.

Brown, M., Hentschel, N., Hannes, M. Stix, H. (2020). Financial innovation, payment choice and cash demand - casual evidence from the staggered introduction of contactless debit cards. University of St. Gallen, Working Papers on Finance n. 2020/02.

Carbo-Valverde, S. and F. Rodriguez-Fernandez (2014). ATM withdrawals, debit card transactions at the point of sale and the demand for currency, SERIEs, 5, 399-417.

Carboni, A., C. Doria and S. Zappa (2020). La produzione statistica nell'emergenza COVID19: la stima dei "viaggi" in bilancia dei pagamenti, Banca d'Italia, Note COVID-19, 19 giugno 2020. 
Carvalho, V. M., Hansen, S., Ortiz, Á., Ramón García, J., Rodrigo, T., Rodriguez Mora, S., Ruiz, J. (2020). Tracking the COVID-19 Crisis with High-Resolution Transaction Data, CEPR Discussion Papers n. 14642.

Chen, H, Qian, W., Wen, Q. (2020). The impact of the COVID-19 pandemic on consumption: learning from high-frequency transaction data. Manuscript available at http://dx.doi.org/10.2139/ssrn.3568574

Ching, A.T., Hayashi, F. (2010) Payment Card Rewards Programs and Consumer Payment Choice. Journal of Banking \& Finance, 34, 1773-87.

Choi, C.Y., Chudik, A. (2019). Estimating impulse response functions when the shock series is observed. Economics Letters, 180, 71-75.

De Livera, A.M., R.J. Hyndman, R. D. Snyder (2011). Forecasting time series with complex seasonal patterns using exponential smoothing. Journal of the American Statistical Association, 106, 1513-1527.

Delle Monache, D., Emiliozzi, S., Nobili, A. (2020). Tracking economic growth during the COVID-19: a weekly indicator for Italy. Bank of Italy, paper presented at the ECB Workshop Tracking the economy with high frequency data, 15-16 October 2020.

Esselink, H. and L. Hernandez (2017). The use of cash by households in the euro area. ECB Occasional Paper Series, No. 201, November.

European Central Bank (2020). Report on a digital euro. October 2020. European Central Bank, available at https://www.ecb.europa.eu/euro/html/digitaleuroreport.en.html

European Central Bank (2020). Report on the Study on the payment attitudes of consumers in the euro area (SPACE), 2020.

Fisher B., P. Kohler and F. Seitz (2004). The demand for euro area currencies: past, present and future. ECB Working Paper Series, No. 330.

Greene, C., \& Stavins, J. (2017). Did the Target data breach change consumer assessments of payment card security? Journal of Payments Strategy \& Systems, 11(2), 121-133.

Jobst, C., Stix, H. (2017). Doomed to Disappear? The Surprising Return of Cash Across Time and Across Countries. CEPR Discussion Paper No. DP12327.

Jordà, O. (2005). Estimation and Inference of Impulse Responses by Local Projections. American Economic Review, 95, 161-182.

Kahn, C. M., Liñares-Zegarra, J. M., \& Stavins, J. (2017). Are there social spillovers in consumers' security assessments of payment instruments?. Journal of Financial Services Research, 52(1-2), 5-34.

Kilian, L. and Kim, Y. J. (2011). How Reliable Are Local Projection Estimators of Impulse Responses? Review of Economics and Statistics, 93(4), 1460-1466.

Kilian, L., Lütkepohl, H. (2017). Structural Vector Autoregressive Analysis. Cambridge University Press.

Klee, E. (2008). How people pay: evidence from grocery store data. Journal of Monetary Economics, 55, 526-541.

Kosse, A. (2013). The Safety of Cash and Debit Cards: A Study on the Perception and Behavior of Dutch Consumers. International Journal of Central Banking, 9(4), 77-98. 
Li, B.G., McAndrews, J., Zhu, W (2019). Two-sided market, R\&D, and payments system evolution. Journal of Monetary Economics, forthcoming.

Lippi, F., A Secchi (2009). Technological change and the households' demand for currency. Journal of Monetary Economics, 56(2), 222-230.

Nakamura, E., Steinsson, J. (2018). Identification in Macroeconomics. Journal of Economic Perspectives, 32, 59-86.

Panetta, F. (2020). Beyond monetary policy-protecting the continuity and safety of payments during the coronavirus crisis. European Central Bank, blog post available at https://www.ecb.europa.eu/press/blog/date/2020/html/ecb.blog200428 328d7ca065.en $\underline{\text { html }}$

Plagborg-Møller, M., Wolf, C.K. (2020). Working Papers. Local Projections and VARs Estimate the Same Impulse Responses. Princeton University, Manuscript.

Ramey, V. A. (2016). Macroeconomic Shocks and Their Propagation. In J. B. Taylor and H. Uhlig (Eds.), Elsevier, Handbook of Macroeconomics, 71-162.

Rinaldi, R. (2017). The role of cash in payment transactions. Bank of Italy, speech available at https://www.bancaditalia.it/pubblicazioni/interventi-vari/int-var2017/en_Rinaldi_22112017.pdf?language_id=1

Rocco, G. (2019). The use of cash in Italy: evidence from the ECB Study on the use of cash by households. Bank of Italy, Occasional Papers n. 481.

Rogoff, K.S. (2016). The Curse of Cash. Economics Books. Princeton University Press.

Rysman, M., \& Schuh, S. (2017). New innovations in payments. Innovation Policy and the Economy, 17(1), 27-48.

Schuh, S., J. Stavins. (2015). How Do Speed and Security Influence Consumers' Payment Behavior? Contemporary Economic Policy, doi: 10.1111/coep.12163.

Simon, J., K. Smith, and T. West. (2010). Price Incentives and Consumer Payment Behavior, Journal of Banking and Finance, 34, 1759-1772.

Stavins, J. (2017). How Do Consumers Make Their Payment Choices? Federal Reserve Bank of Boston, Research Data Reports n. 17-1.

Stix, H. (2004). How do debit cards affect cash demand? Survey data evidence. Empirica, 31(2), 93-115.

Stix, H. (2013). Why do People Save in Cash? Distrust, Memories of Banking Crises, Weak Institutions and Dollarization. Journal of Banking \& Finance, 37, 4087-4106.

Stock, J. H. and Watson, M. W. (2018). Identification and Estimation of Dynamic Causal Effects in Macroeconomics Using External Instruments. Economic Journal, 128, 917-948.

Visco, I. (2020). Bank of Italy. The Governor's Concluding Remarks for 2019, available at https://www.bancaditalia.it/pubblicazioni/interventi-governatore/integov2020/encf_2019.pdf?language id=1

Wakamori, N., \& Welte, A. (2017). Why do shoppers use cash? Evidence from shopping diary data. Journal of Money, Credit and Banking, 49(1), 115-169. 


\section{List of Figures}

Figure 1: indicators of the pandemic intensity

(daily data; index maximum value $=100$ )

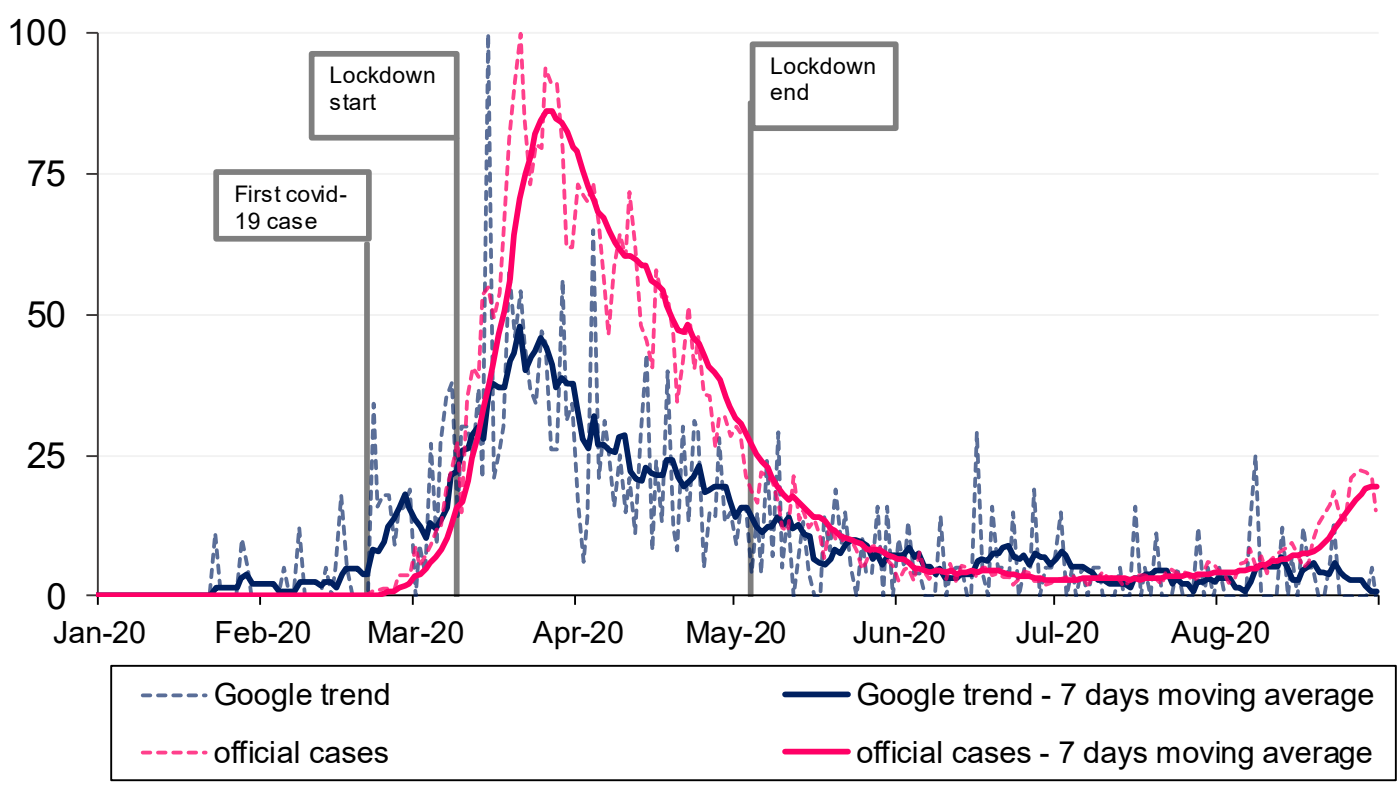

Figure 2: Use of cards at POS and ATMs

(daily data; millions of $€$ )

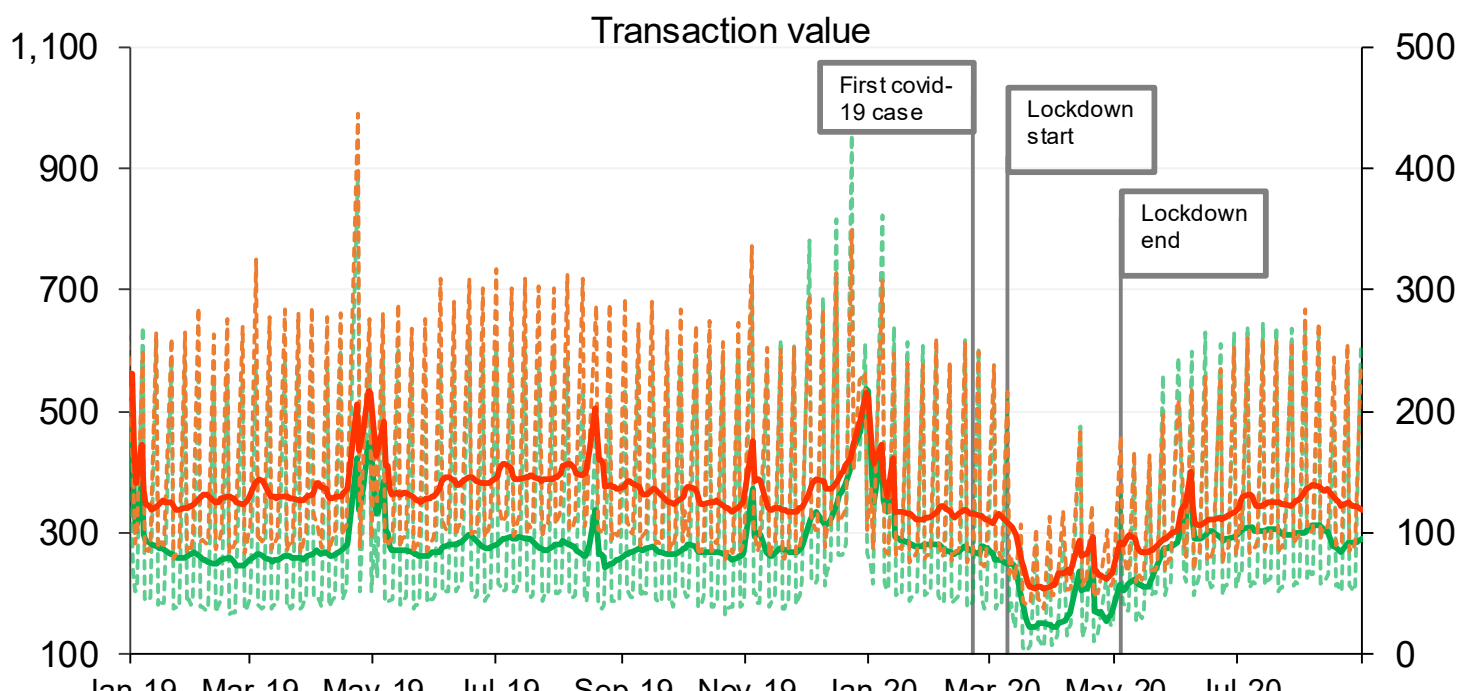

Jan-19 Mar-19 May-19 Jul-19 Sep-19 Nov-19 Jan-20 Mar-20 May-20 Jul-20

\begin{tabular}{|ll|}
\hline$-\cdots$ POS (Ih axis) & POS -5 days moving average (Ih axis) \\
$-\cdots$ & ATMs -5 days moving average (rh axis) \\
\hline
\end{tabular}


Figure 3: Indicators of payment habits based on debit cards (daily data)
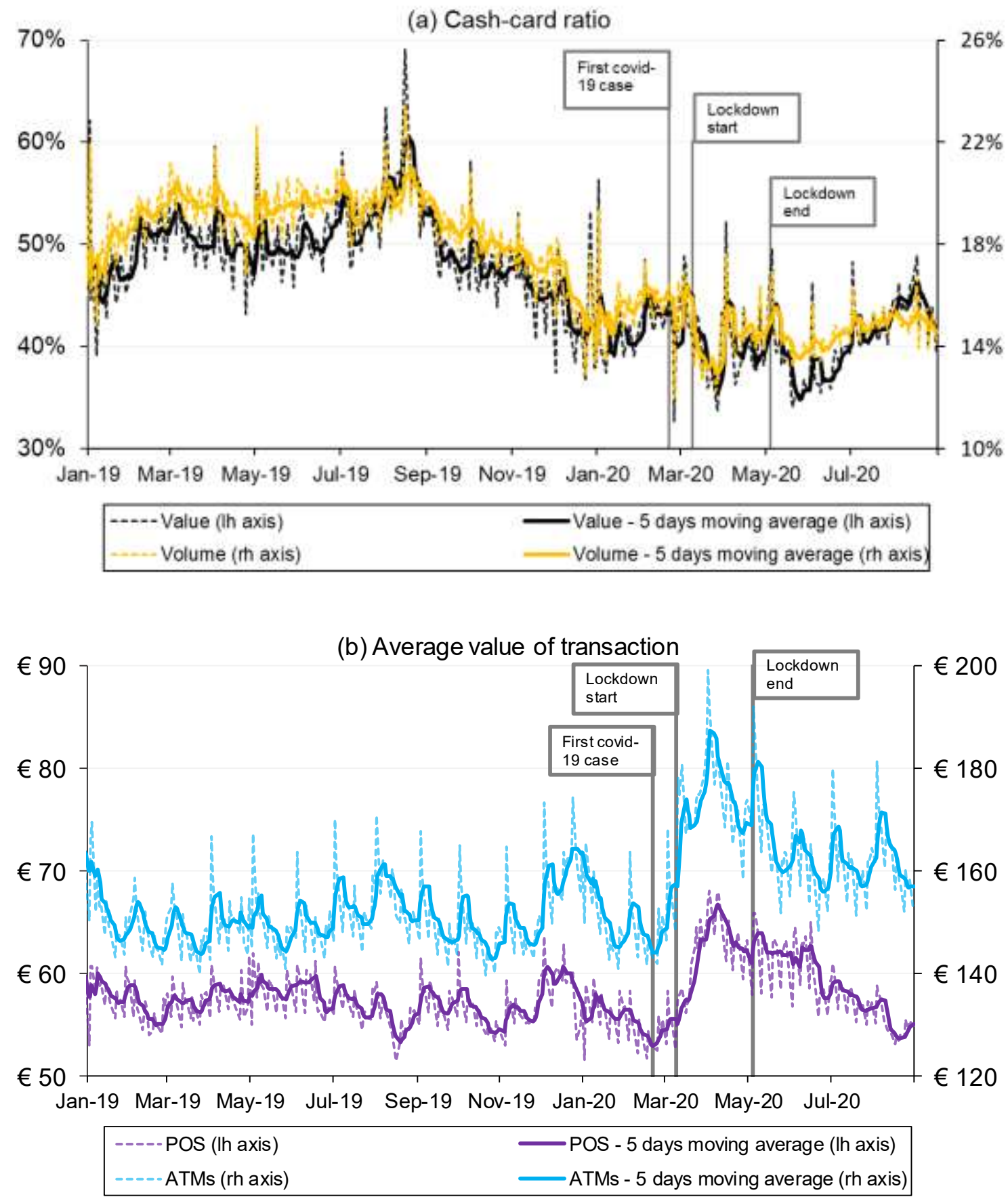
Figure 4: Indicators of payment habits based on credit cards (daily data)
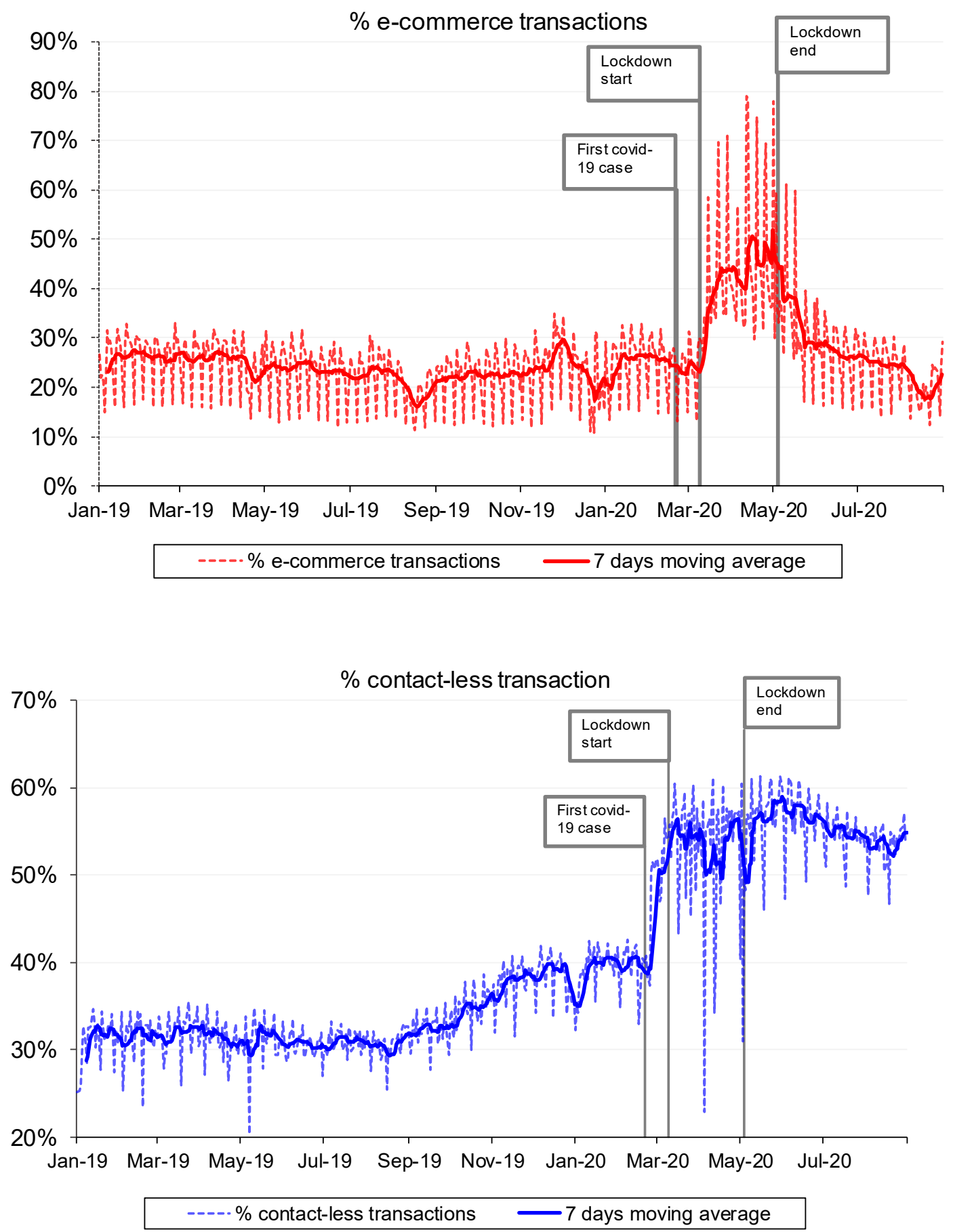
Figure 5. Effects of the pandemic on payment habits
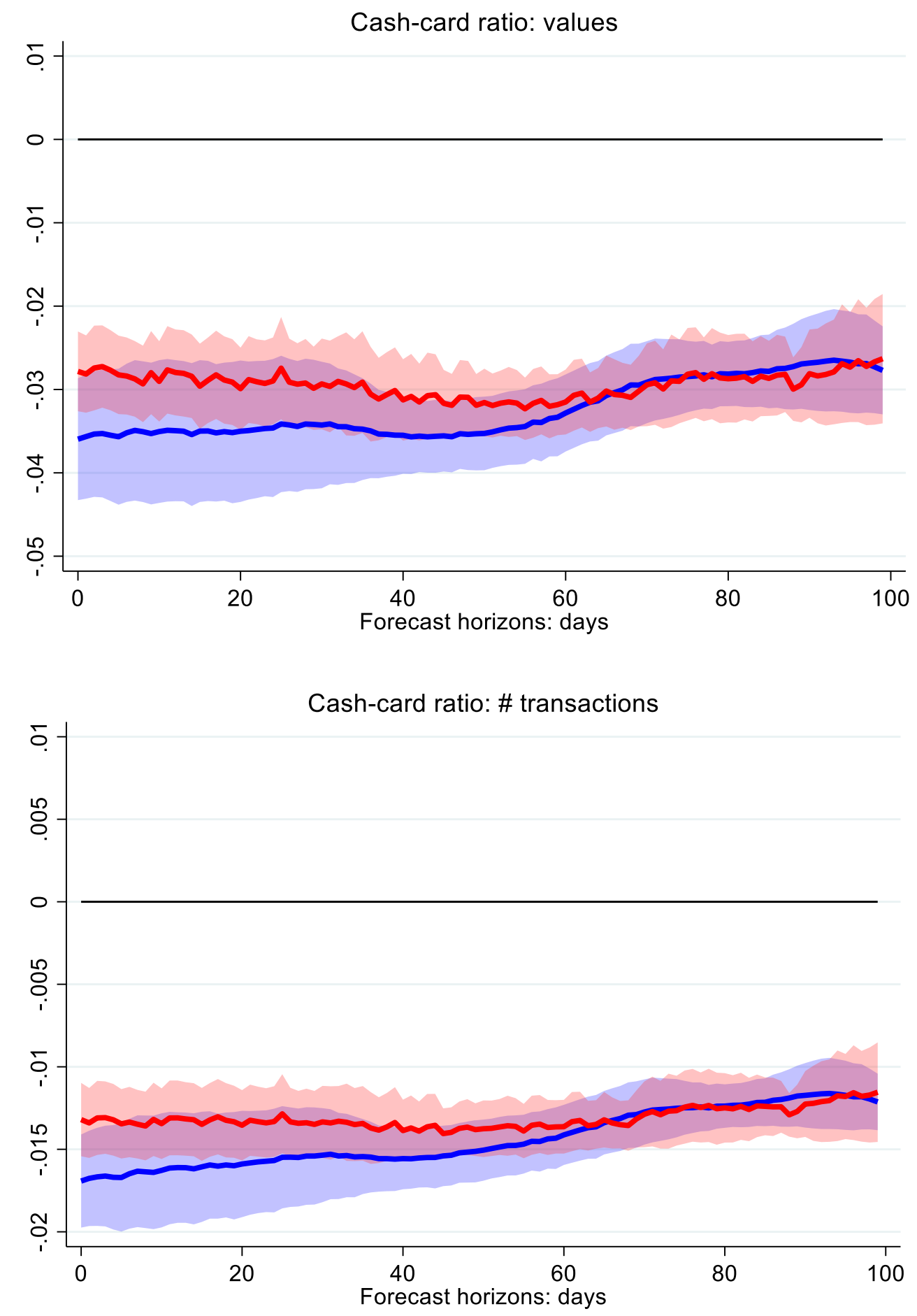

Notes: the red line refers to impulse response functions to a pandemic shock, as captured by a Google Trend indicator; the blue line refers to impulse response functions to a pandemic shock, as captured by official new COVID-19 cases. Authors' elaborations on data from BI-COMP and card processors. 
Figure 5 (continues). Effects of the pandemic on payment habits
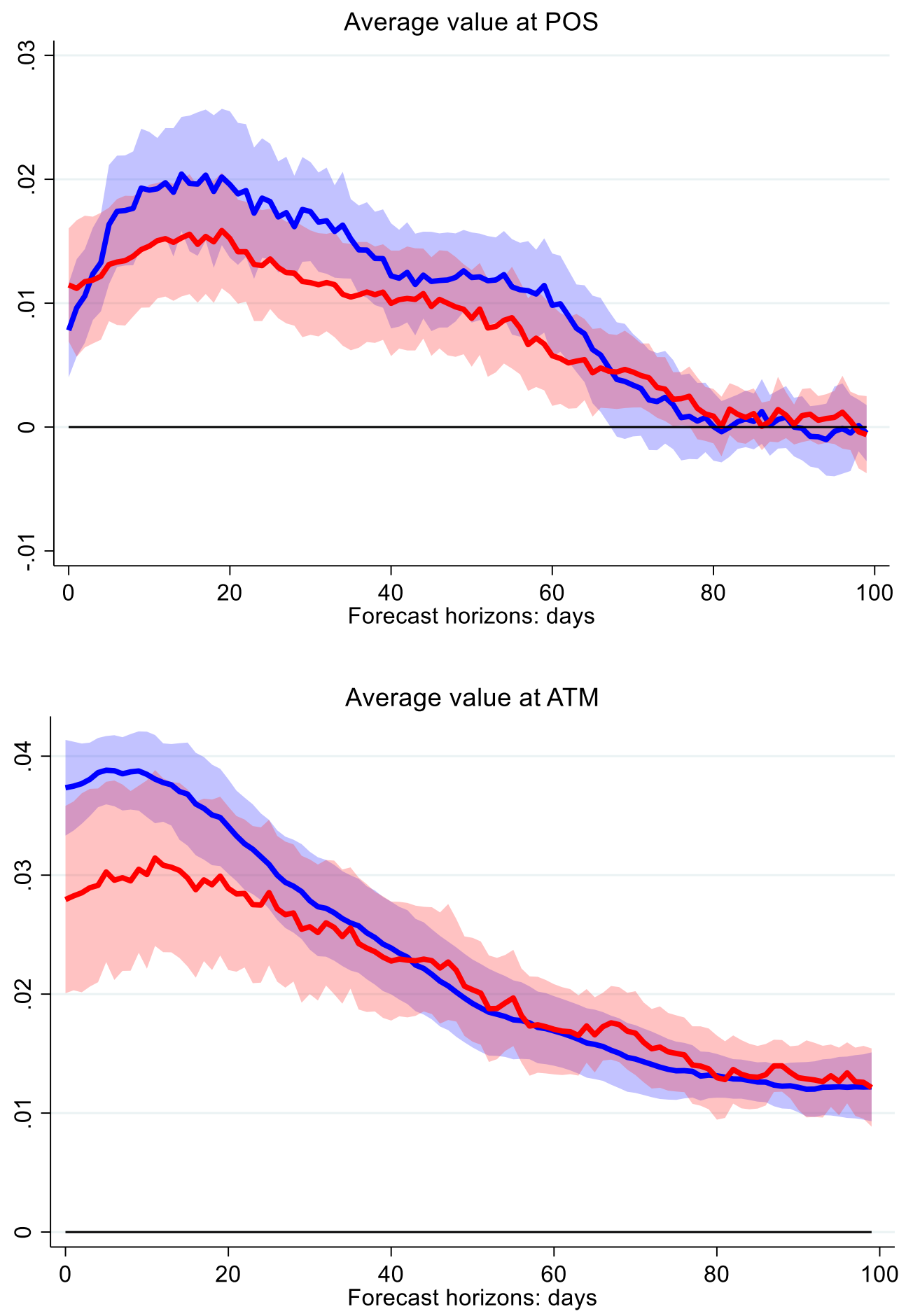

Notes: the red line refers to impulse response functions to a pandemic shock, as captured by a Google Trend indicator; the blue line refers to impulse response functions to a pandemic shock, as captured by official new COVID-19 cases. Authors' elaborations on data from BI-COMP and card processors. 
Figure 5 (continues). Effects of the pandemic on payment habits
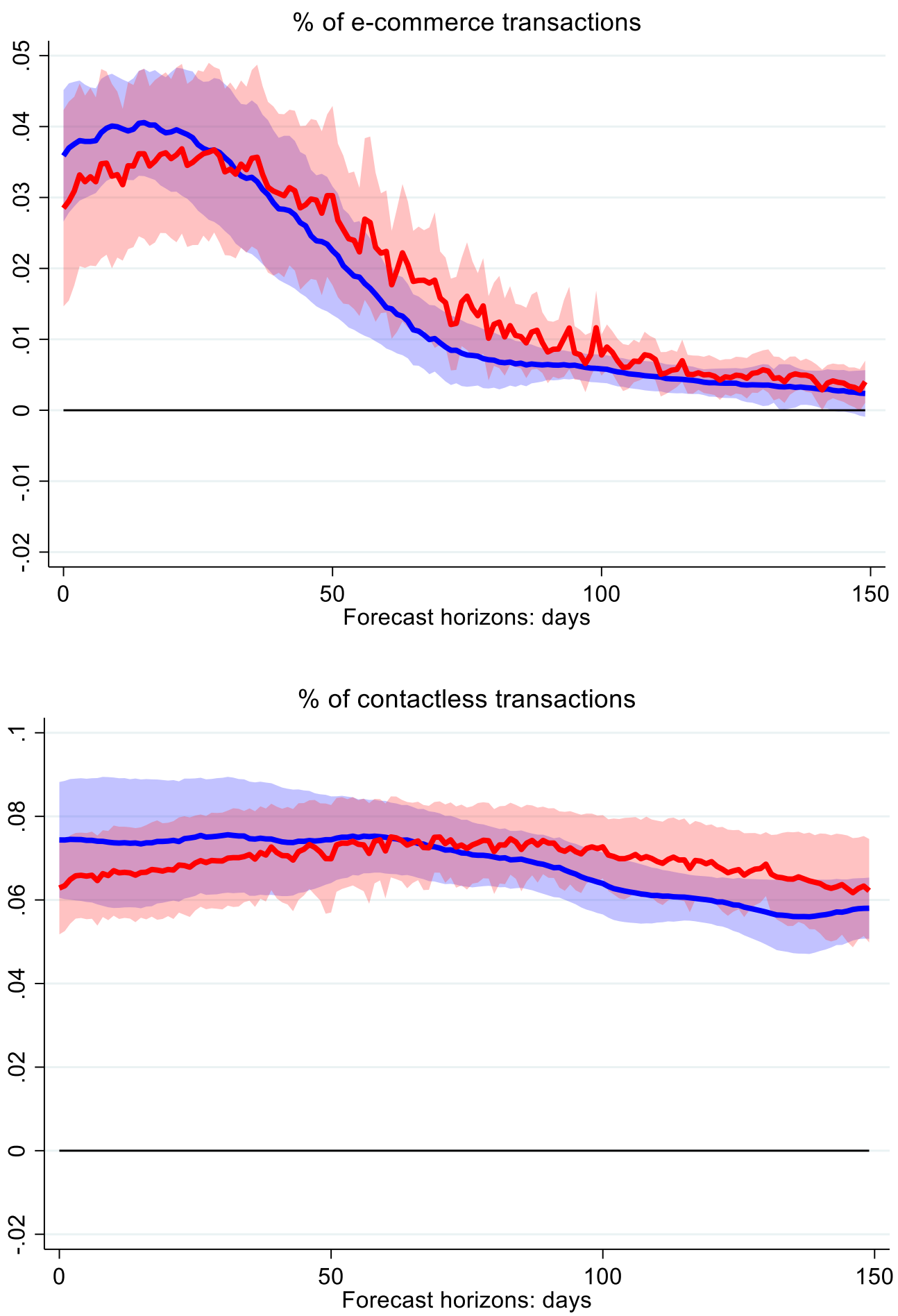

Notes: the red line refers to impulse response functions to a pandemic shock, as captured by a Google Trend indicator; the blue line refers to impulse response functions to a pandemic shock, as captured by official new COVID-19 cases. Authors' elaborations on data from BI-COMP and card processors. 
Figure 6. Developments in circulation and its main components (daily data; billions of $\epsilon$ )
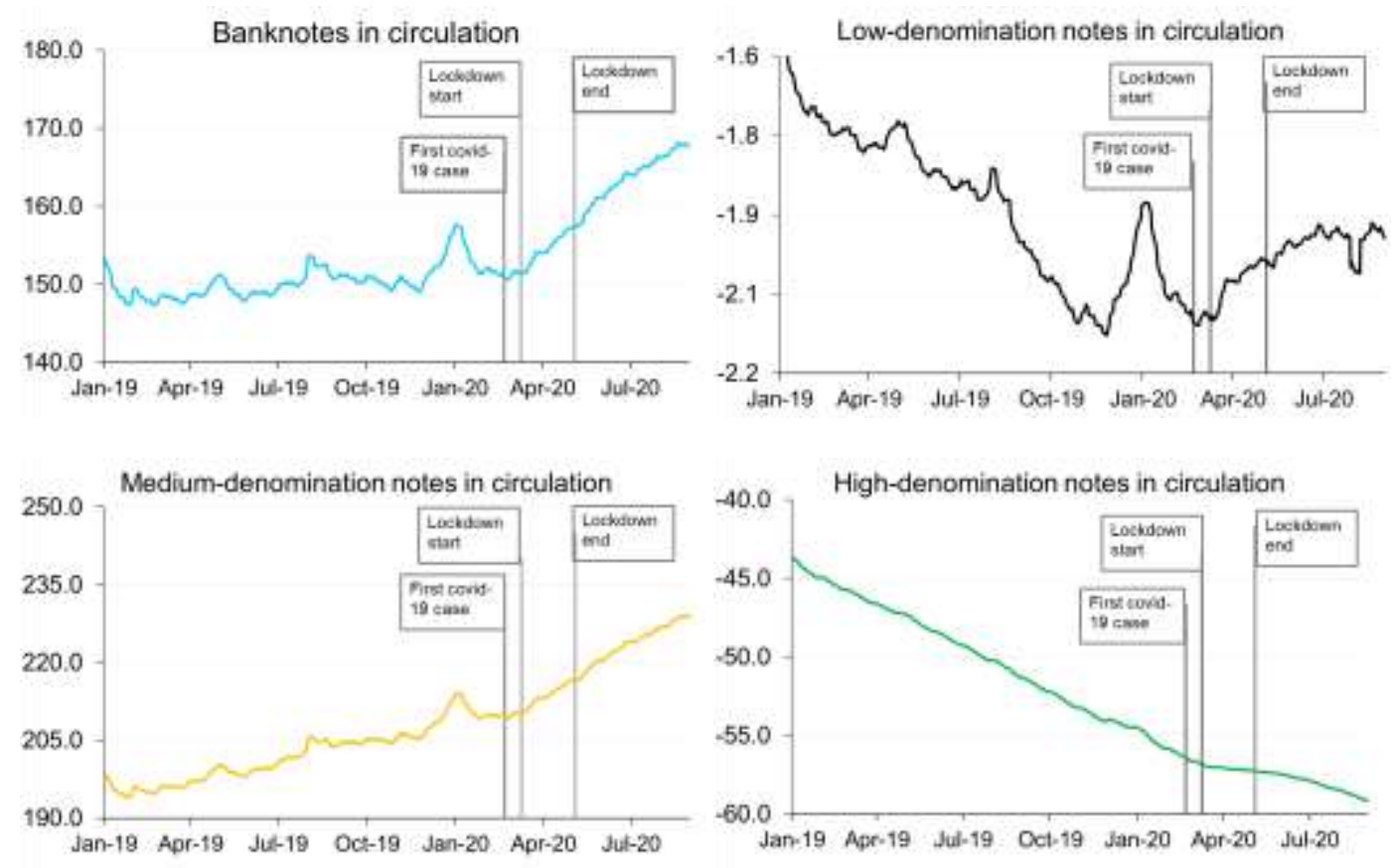

Figure 7. Effects of the pandemic on banknotes in circulation
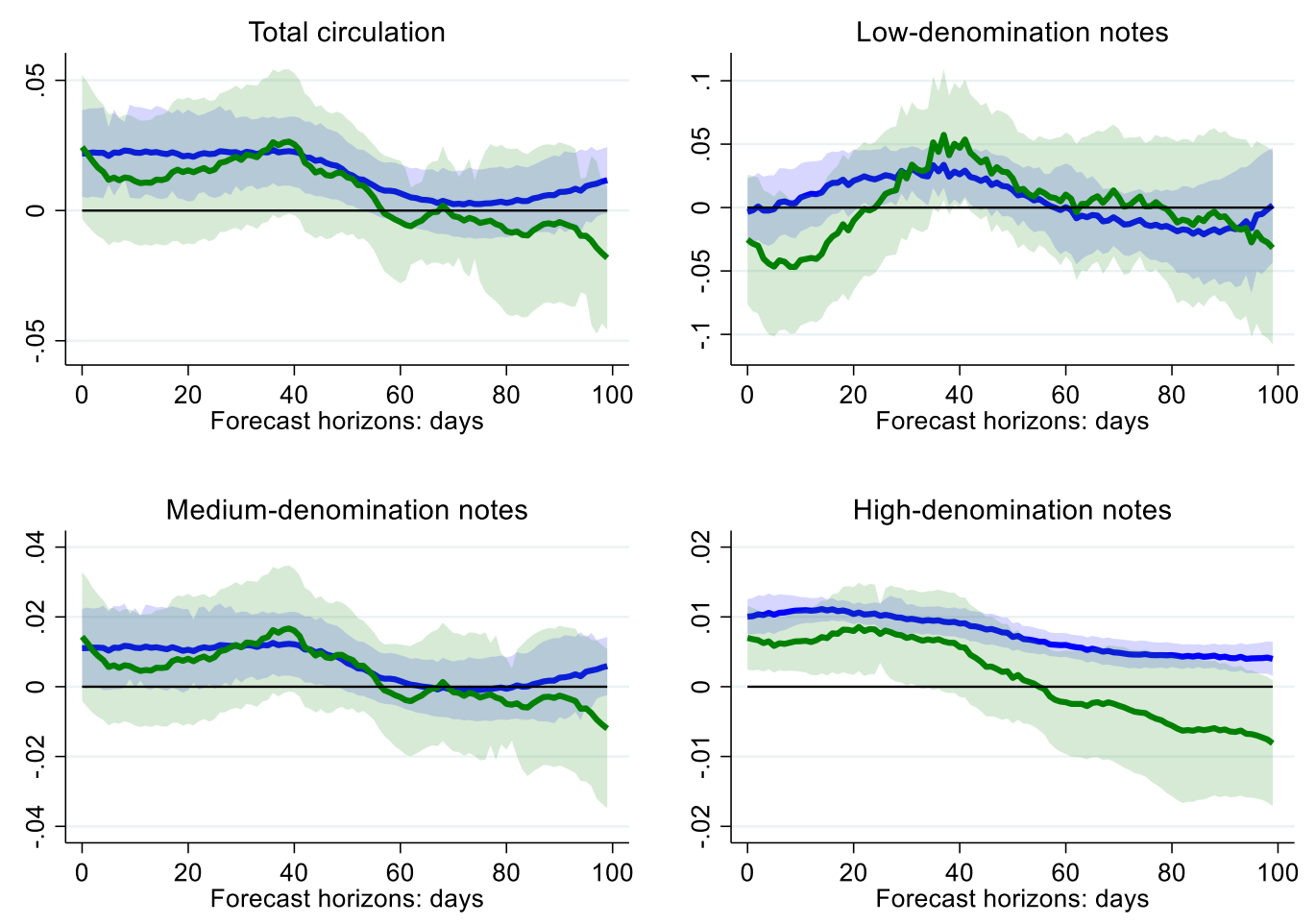

Unadjusted

Adjusted for foreign flows 


\section{Appendix A. Effects of the pandemic on other variables}

Figure A1. Effects of the pandemic on POS \& ATM variables
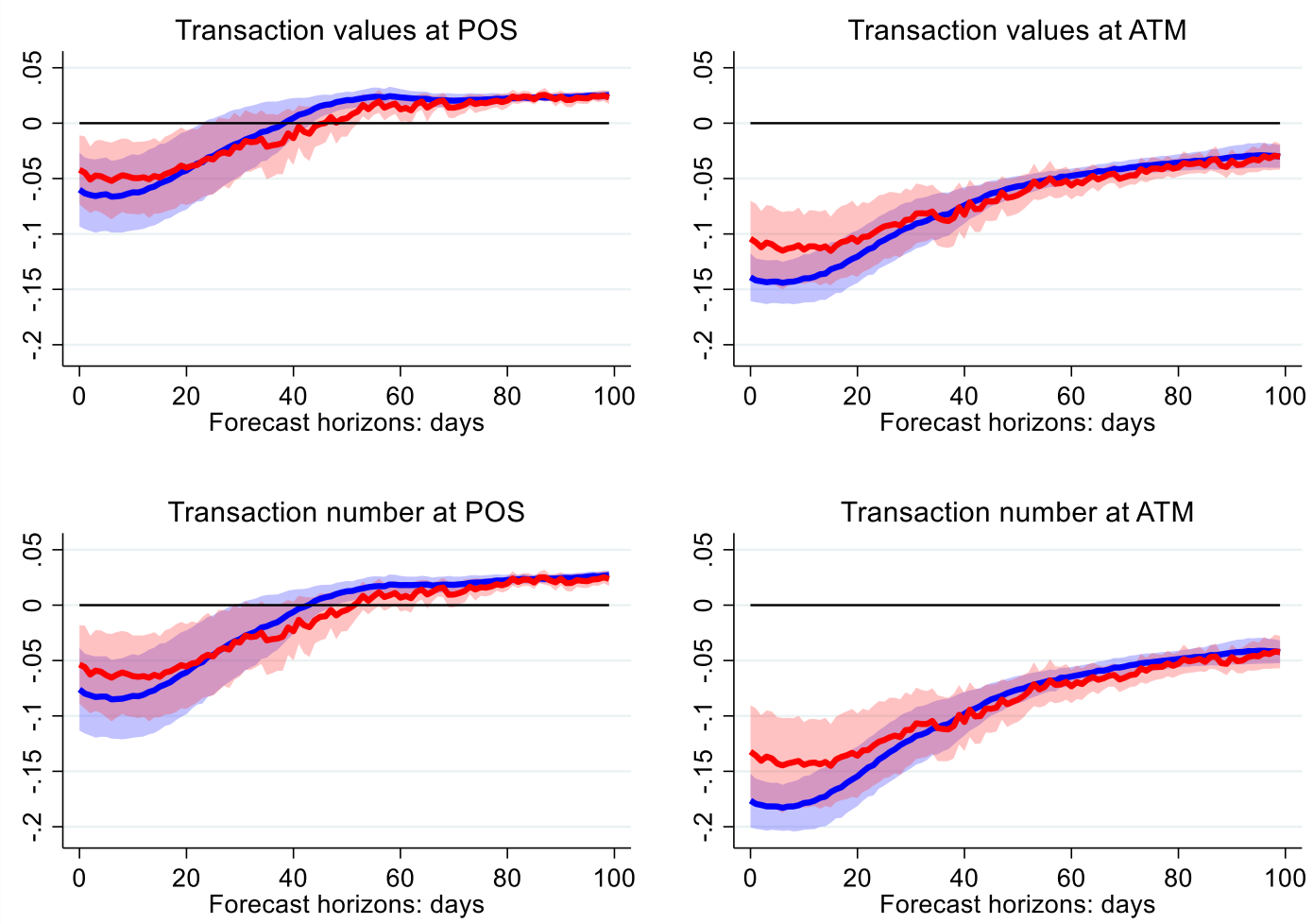

Figure A2. Effects of the pandemic on card transaction values
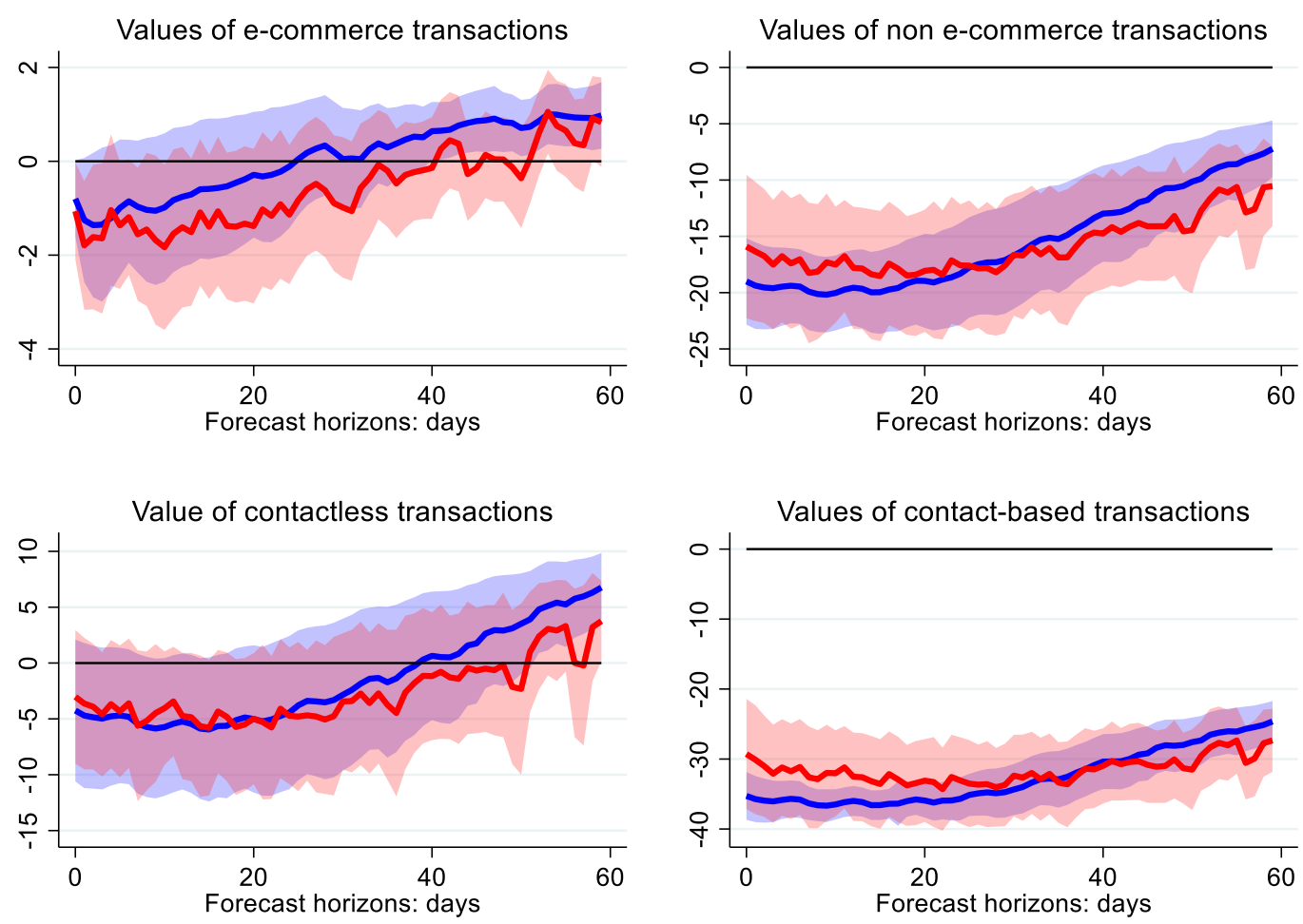
Figure A3. Effects of the pandemic on withdrawals of banknotes
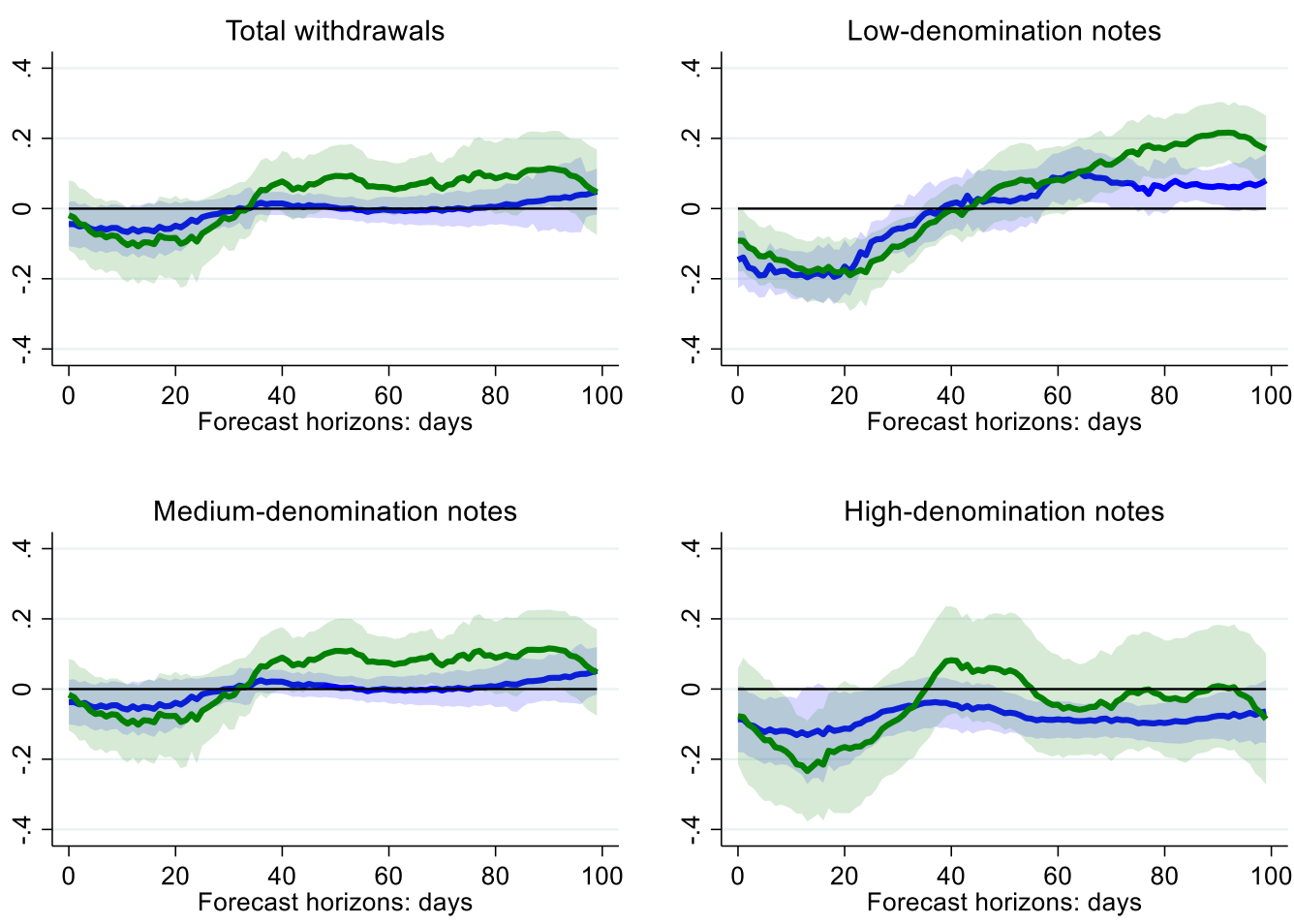

Unadjusted

Adjusted for foreign flows

Figure A4. Effects of the pandemic on lodgements of banknotes
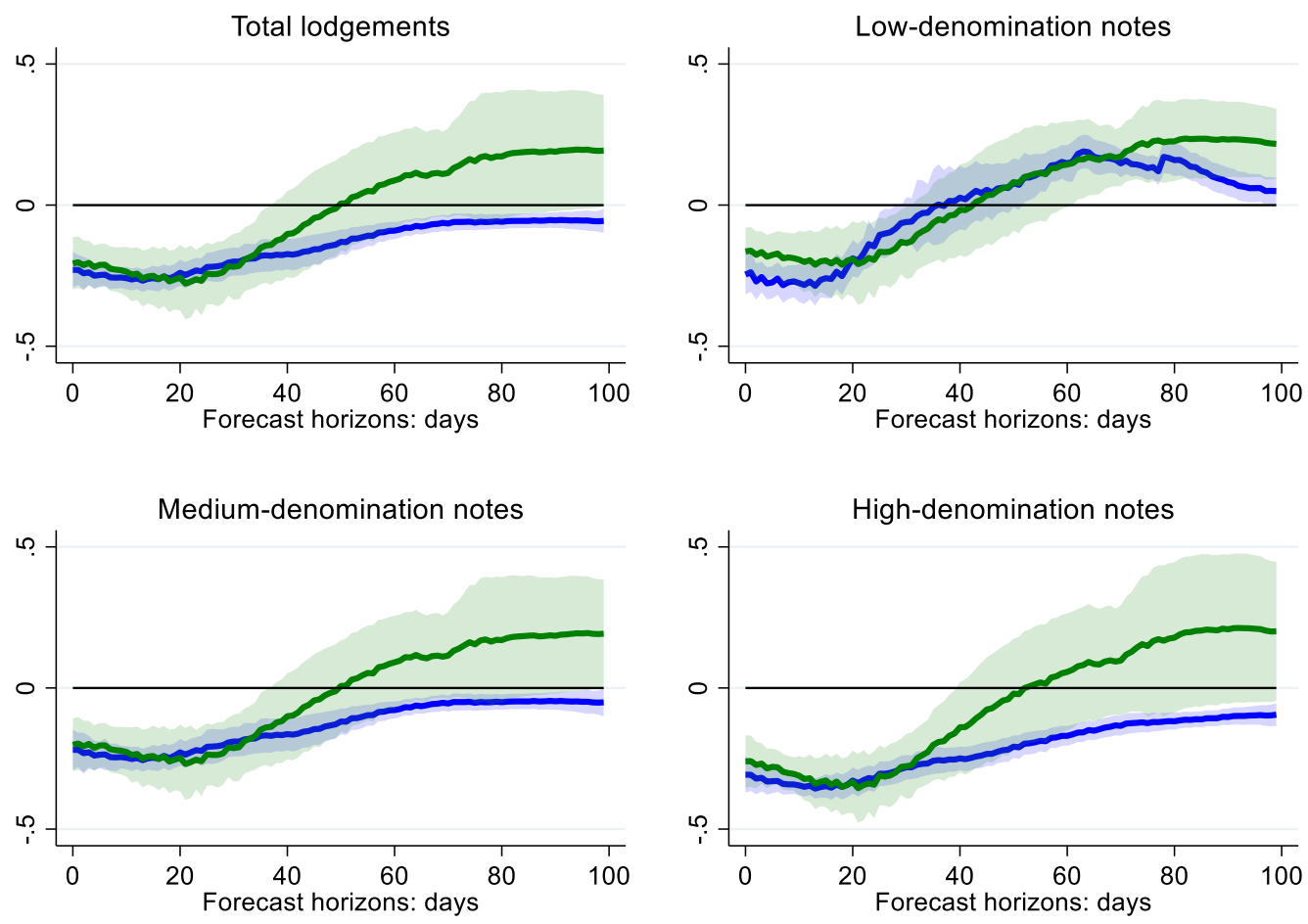


\section{Appendix B. Figures relating to robustness analysis}

\section{Figure B1. Estimated effects with rolling samples}
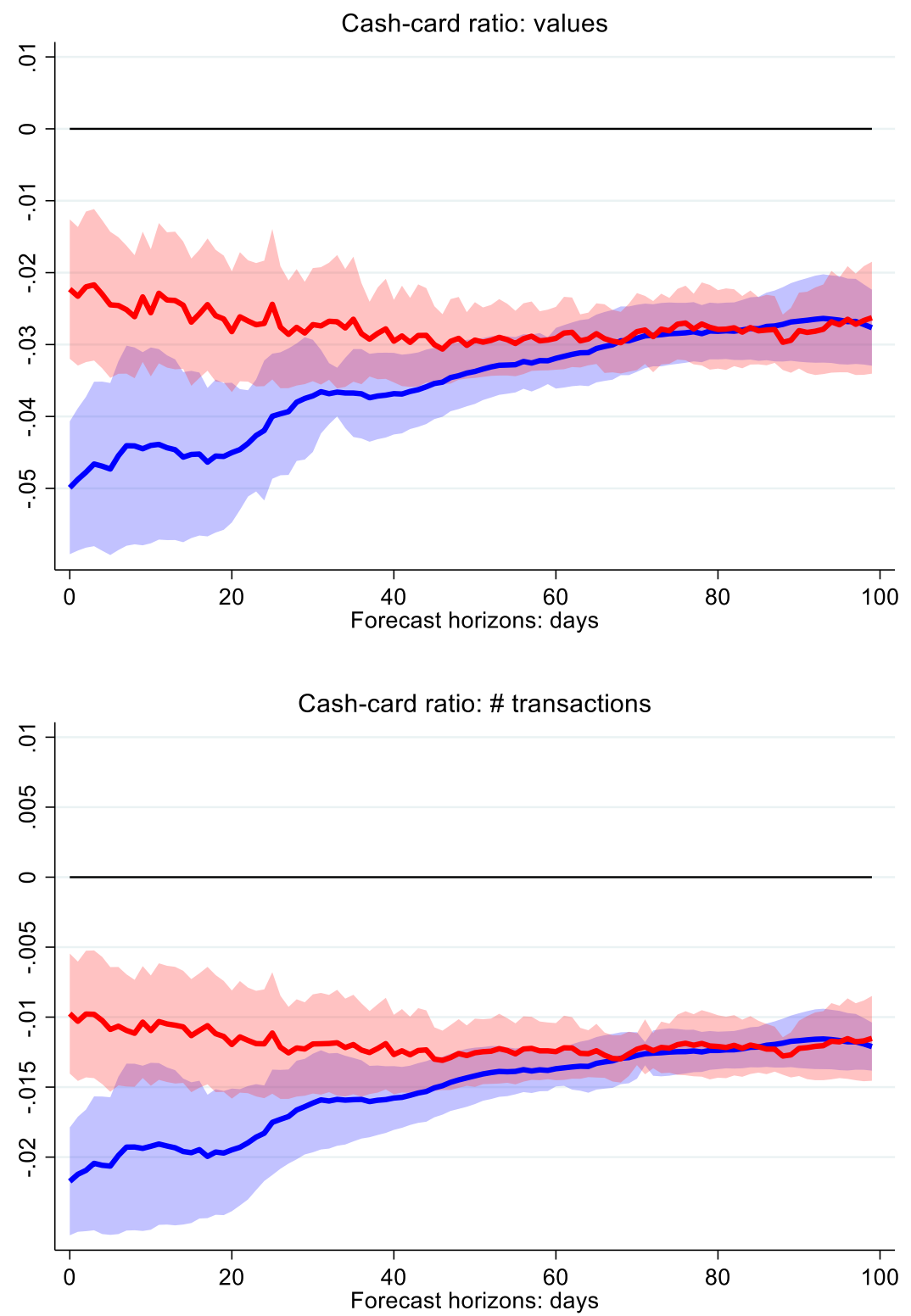

Notes: the red line refers to impulse response functions to a pandemic shock, as captured by a Google Trend indicator; the blue line refers to impulse response functions to a pandemic shock, as captured by official new COVID-19 cases. Authors' elaborations on data from BI-COMP and card processors. 


\section{Figure B1 (continues). Estimated effects with rolling samples}
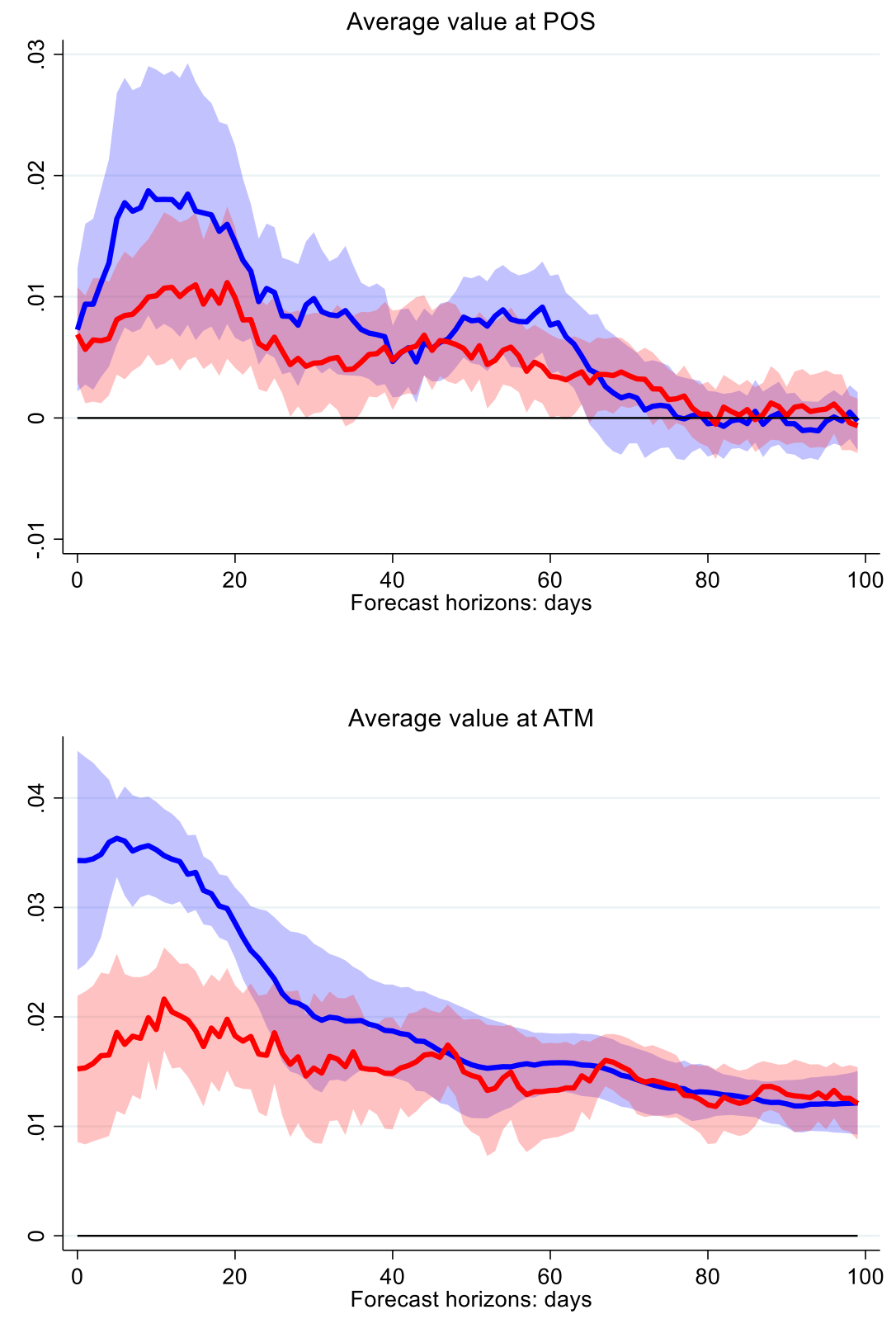

Notes: the red line refers to impulse response functions to a pandemic shock, as captured by a Google Trend indicator; the blue line refers to impulse response functions to a pandemic shock, as captured by official new COVID-19 cases. Authors' elaborations on data from BI-COMP and card processors. 


\section{Figure B1 (continues). Estimated effects with rolling samples}
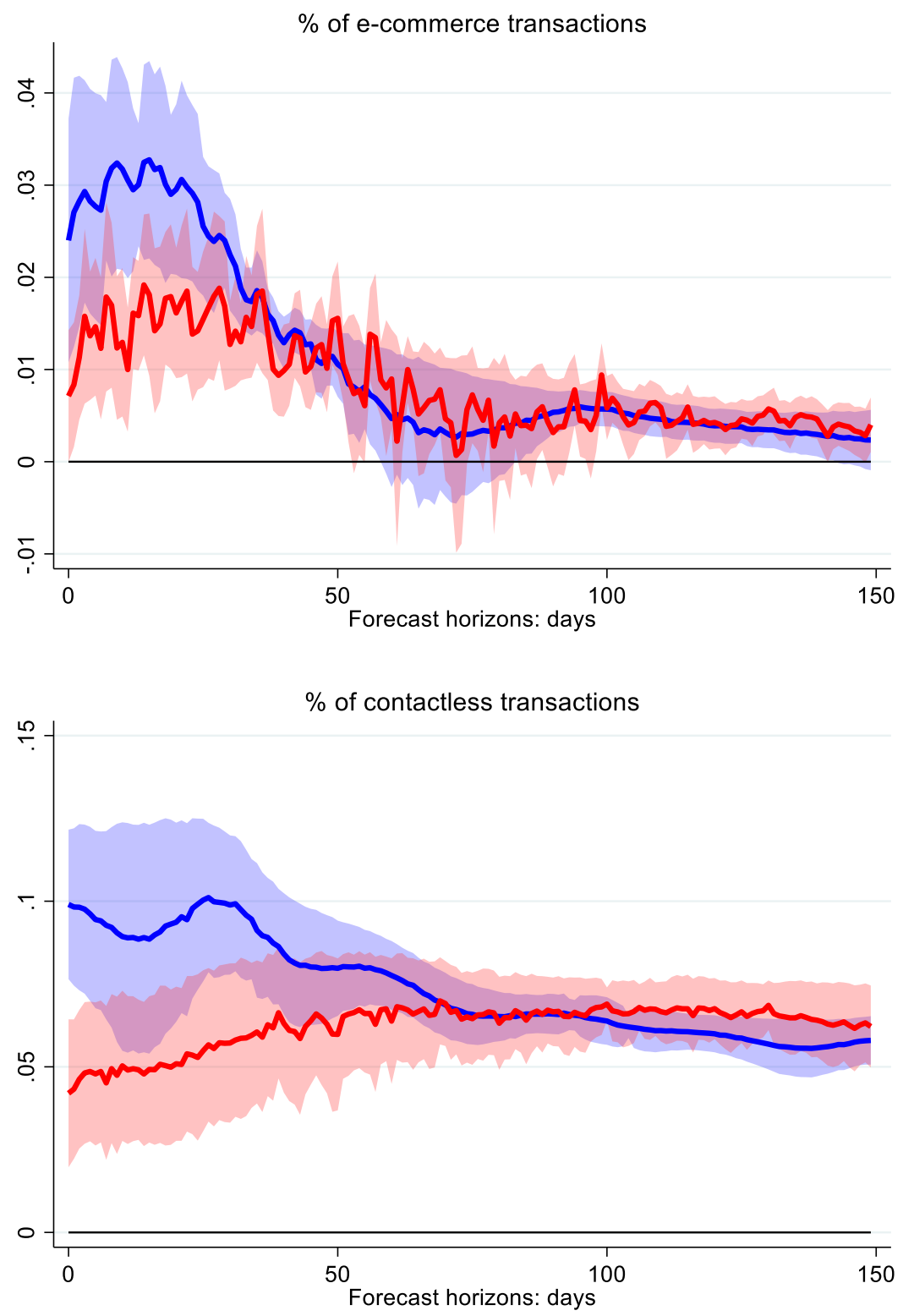

Notes: the red line refers to impulse response functions to a pandemic shock, as captured by a Google Trend indicator; the blue line refers to impulse response functions to a pandemic shock, as captured by official new COVID-19 cases. Authors' elaborations on data from BI-COMP and card processors. 
Figure B1 (continues). Estimated effects with rolling samples
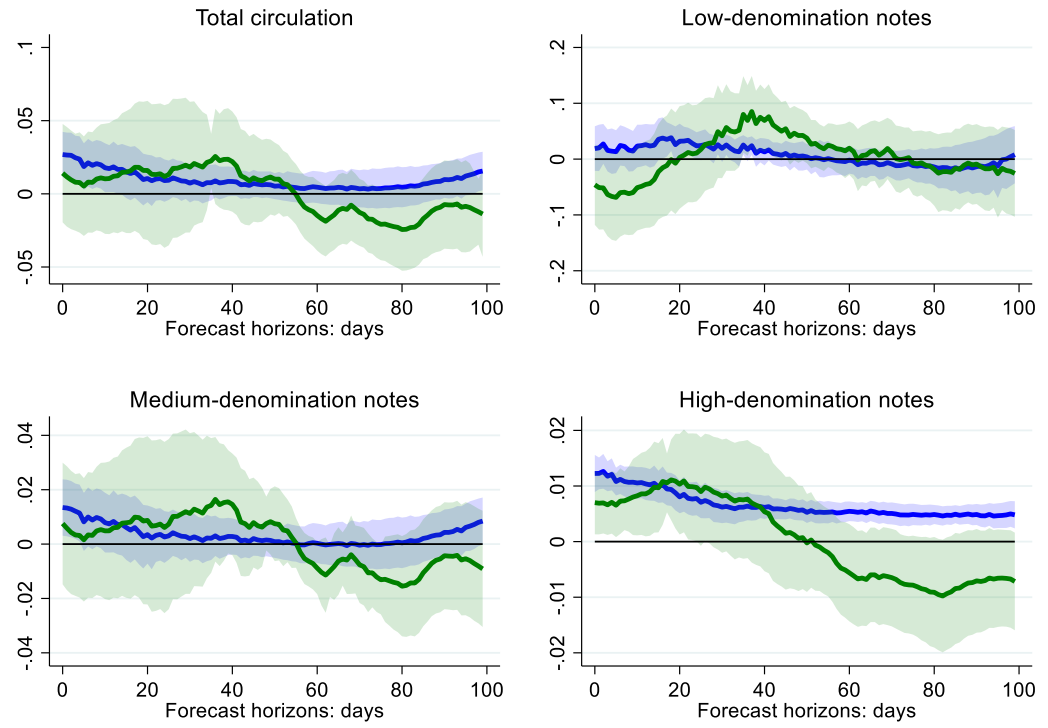

Figure B2. Effects on share of contactless transactions in grocery sector

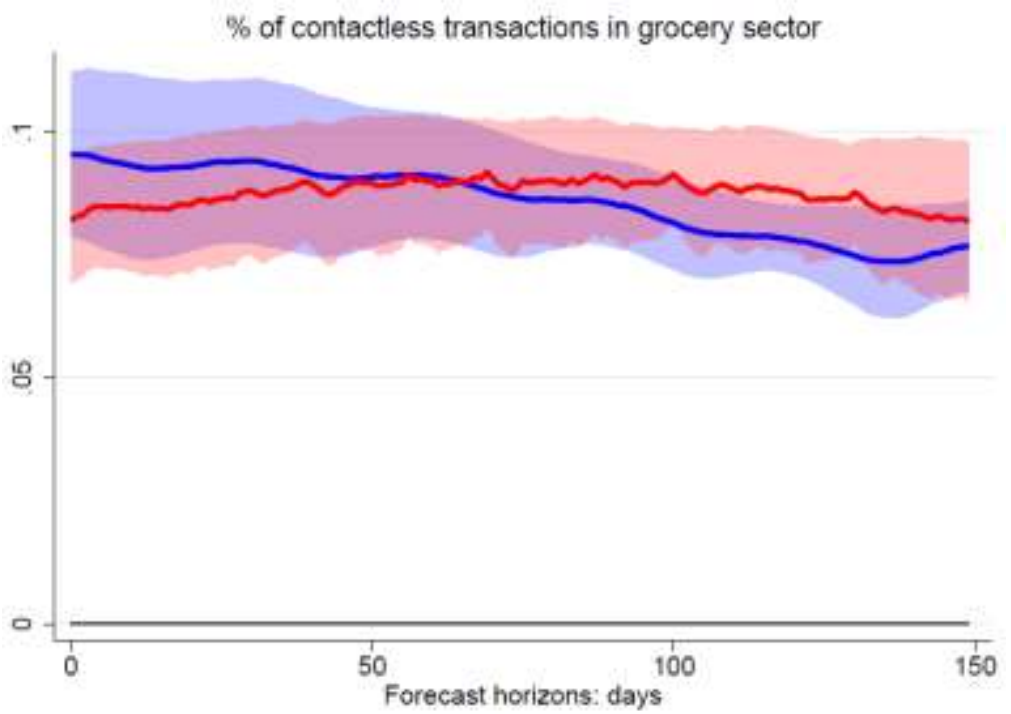

